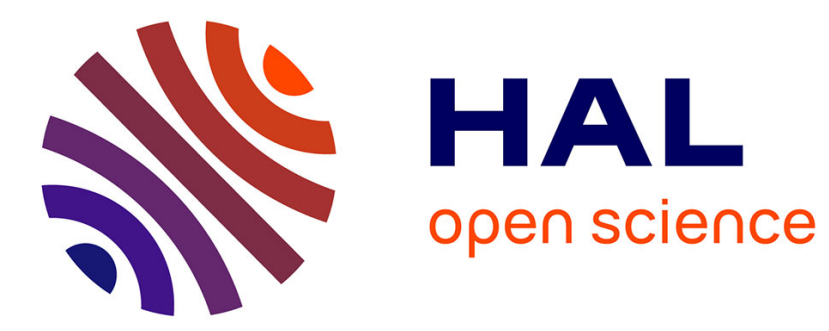

\title{
The Chamberlin-Courant Rule and the k-Scoring Rules: Agreement and Condorcet Committee Consistency
}

\author{
Mostapha Diss, Eric Kamwa, Abdelmonaim Tlidi
}

\section{To cite this version:}

Mostapha Diss, Eric Kamwa, Abdelmonaim Tlidi. The Chamberlin-Courant Rule and the k-Scoring Rules: Agreement and Condorcet Committee Consistency. 2018. halshs-01817943

\section{HAL Id: halshs-01817943 \\ https://shs.hal.science/halshs-01817943}

Preprint submitted on 18 Jun 2018

HAL is a multi-disciplinary open access archive for the deposit and dissemination of scientific research documents, whether they are published or not. The documents may come from teaching and research institutions in France or abroad, or from public or private research centers.
L'archive ouverte pluridisciplinaire HAL, est destinée au dépôt et à la diffusion de documents scientifiques de niveau recherche, publiés ou non, émanant des établissements d'enseignement et de recherche français ou étrangers, des laboratoires publics ou privés. 
UMR 5824

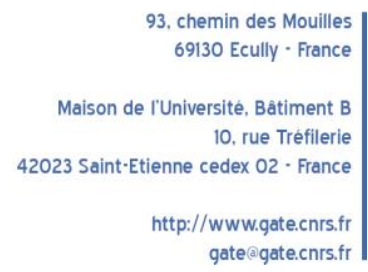

WP 1812 - June 2018

\title{
The Chamberlin-Courant Rule and the k-Scoring Rules: Agreement and Condorcet Committee Consistency
}

\author{
Mostapha Diss, Eric Kamwa, Abdelmonaim Tlidi
}

\begin{abstract}
:
For committee or multiwinner elections, the Chamberlin-Courant rule (CCR), which combines the Borda rule and the proportional representation, aims to pick the most representative committee (Chamberlin and Courant, 1983). Chamberlin and Courant (1983) have shown that if the size of the committee to be elected is $k=1$ among $m \geq 3$ candidates, the CCR is equivalent to the Borda rule; Kamwa and Merlin (2014) claimed that if $k=m-1$, the CCR is equivalent to the $k$-Plurality rule. In this paper, we explore what happens for $1<k<m-1$ by computing the probability of agreement between the CCR and four $k$ scoring rules: $k$-Plurality, $k$-Borda, $k$-Negative Plurality and Bloc. Our results show that for committees of at least two members, the CCR usually leads to a committee recommended by the $k$-Plurality rule. Furthermore, we evaluate the probability of the CCR to select the Condorcet committee à la Gehrlein when it exists. The Condorcet committee à la Gehrlein is a fixed size subset of candidates such that every meber defeats every non-member in pairwise comparisons. In this matter, our results indicate that the CCR performs less well than the $k$-Borda rule and the Bloc rule but better than the $k$-Plurality and the $k$ Negative Plurality rules.
\end{abstract}

\section{Keywords:}

Committee, Representativeness, Borda, Condorcet, Chamberlin-Courant, $k$-Scoring rule

JEL codes:

D71, D72

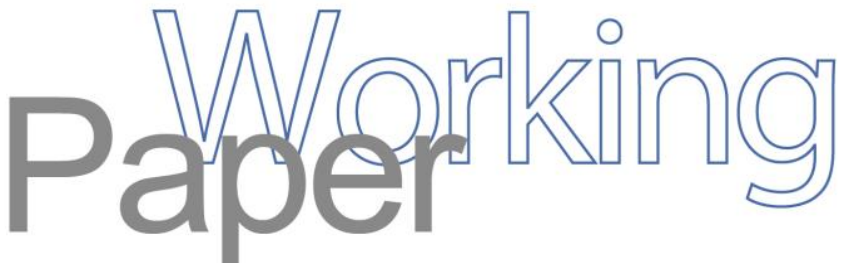




\title{
The Chamberlin-Courant Rule and the $k$-Scoring Rules: Agreement and Condorcet Committee Consistency*
}

\author{
Mostapha Diss ${ }^{\dagger}$
}

\author{
Eric Kamwa
}

March 6, 2018

\author{
Abdelmonaim Tlidi ${ }^{\S}$
}

\begin{abstract}
For committee or multiwinner elections, the Chamberlin-Courant rule (CCR), which combines the Borda rule and the proportional representation, aims to pick the most representative committee (Chamberlin and Courant, 1983). Chamberlin and Courant (1983) have shown that if the size of the committee to be elected is $k=1$ among $m \geq 3$ candidates, the CCR is equivalent to the Borda rule; Kamwa and Merlin (2014) claimed that if $k=m-1$, the CCR is equivalent to the $k$-Plurality rule. In this paper, we explore what happens for $1<k<m-1$ by computing the probability of agreement between the CCR and four $k$-scoring rules: $k$-Plurality, $k$-Borda, $k$-Negative Plurality and Bloc. Our results show that for committees of at least two members, the CCR usually leads to a committee recommended by the $k$-Plurality rule. Furthermore, we evaluate the probability of the CCR to select the Condorcet committee à la Gehrlein when it exists. The Condorcet committee $\grave{a}$ la Gehrlein is a fixed size subset of candidates such that every member defeats every non-member in pairwise comparisons. In this matter, our results indicate that the CCR performs less well than the $k$-Borda rule and the Bloc rule but better than the $k$-Plurality and the $k$-Negative Plurality rules.
\end{abstract}

Keywords: Committee, Representativeness, Borda, Condorcet, Chamberlin-Courant, $k$-Scoring rule.

JEL Classification Number: D71, D72

*The authors would like to thank Winfried Bruns for comments and discussions about the volume calculation of some polytopes using the software Normaliz. Early drafts of this paper have been presented at CREM seminar in Caen Normandy university (December 2017), LED seminar in Paris 8 university (January 2018), and the seminar of the department of Mathematics in Kenitra university, Morocco (January 2018). The authors are grateful for comments received from the participants at all those seminars and conferences.

$\dagger$ Univ Lyon, UJM Saint-Etienne, GATE UMR 5824, F-42023 Saint-Etienne, France. Email: diss@gate.cnrs.fr.

${ }^{\ddagger}$ Université des Antilles, Faculté de Droit et d'Economie de la Martinique and Laboratoire Caribéen de Sciences Sociales LC2S UMR CNRS 8053, F-97275 Schoelcher Cedex. Email: eric.kamwa@univ-antilles.fr.

$\S$ University Cadi Ayyad of Marrakesh, GREER, National School of Applied Science-Safi, Route Sidi Bouzid, B.P. 63, 46000 Safi, Morocco. Email: mtlidi2010@gmail.com. 


\section{Introduction}

In real multiwinner elections, the output of the voters' preferences (votes, rankings) is a subset of the competing candidates. The size of the subset of the winners can be fixed in advance as in committee selection or parliamentary elections. There are a number of different voting methods that can be used in line with the objective pursued; it may be either to draw up a first list of candidates that will be submitted afterwards to deliberation in order to determine a final winner from this list or to select a subset of candidates (a committee) that accurately reflects the electorate.

Since the pioneer works of Dodgson (1884, 1876), Droop (1881), and Sterne (1871), the committee selection framework has been of great interest in the late 80s to numerous research teams among the political scientists, economists and computer scientists. In the social choice literature as in real life, there are many voting rules that can be used for electing committees such as (i)-the constant scoring rules also called $k$-scoring rules, where $k$ refers to the size of the committee to be elected. Under these rules, each candidate gets some points from each voter according to her position in the voter's preference and the candidates with the $k$ highest aggregated scores are elected. We present and define some well-known $k$-scoring rules in Section 2. Without being exhaustive, the following papers deal with this approach: Bock et al. (1998), Debord (1993), Diss and Doghmi (2016), Dummett (1984), Kamwa and Merlin (2015). It is important to mention that Elkind et al. (2017), Skowron et al. (2016) recently examined the properties of some voting rules in multi-winner context and proposed a set of natural properties (axioms) by which these voting rules can be examined. (ii)- the rules based on the Approval Voting under which each voter distinguishes the candidates she approves of from those she considers as unacceptable and the winners are the candidates with the largest number of approvals. In the multiwinner context, there are several approaches dealing with approval voting-based rules. The reader is referred to the works of Aziz et al. (2017), Brams (2008), Brams et al. (2005), Kilgour and Marshall (2012), Kilgour et al. (2006), among others. (iii)- the rules based on the Condorcet principle which elect the subset(s) of candidates such that no member is beaten in pairwise comparisons by any outside contender. To have a quick overview of this family of multiwinner voting rules, the reader may refer to the works of Barberà and Coelho (2008), Elkind et al. (2011, 2015), Fishburn (1981), Gehrlein (1985), Kamwa (2017), Kamwa and Merlin (2018), Kaymak and Sanver (2003), Ratliff (2003), among others.

According to Elkind et al. (2017) and Faliszewski et al. (2011), if the objective pursued is not the shortlisting but is to find a diverse committee (such that its members accurately reflect the whole electorate), the voting rule suggested by Chamberlin and Courant (1983) was designed to fulfill such a purpose. The Chamberlin-Courant rule (here after, CCR) combines the proportional representation and the Borda rule (defined later). Given the size of the committee to be elected and the set of all possible committees, the CCR selects the committee(s) that maximize(s) the representativeness value. For a given committee, the representativeness value is equal to the sum of the Borda weights that voters give to candidates that better represent them in this committee. The more a candidate is better ranked by a voter, the more she represents this voter in such a committee. In Section 2, we 
provide a formal definition of the CCR. The above definition is also known as the utilitarian variant of the CCR; its egalitarian variant has been introduced by Betzler et al. (2013). For more details on the other variant and the links between both versions, the reader is referred to the papers of Betzler et al. (2013), Elkind et al. (2017), Procaccia et al. (2008). In this paper, we focus on the utilitarian variant as originally introduced by Chamberlin and Courant (1983).

Notice that in the recent years, there has been a growing interest on the CCR among the computational social choice scientists concerning its complexity and its various approximations. More particularly, it has been shown that finding the winners under the CCR belongs to the class of NP-hard problems. ${ }^{1}$ In other words, even though the CCR is suited to ensure proportional representation, finding the outcome(s) can be computationally intractable as the number of candidates and the size of the committee to be elected increase. This drawback may compromise the use of this rule in real elections with a huge number of candidates for the benefice of scoring rules. The important question that then arises is the following: Are there scoring rules equivalent to the CCR in the sense that they always lead to the same outcome as the CCR?

A first answer comes from Chamberlin and Courant (1983) as they showed that when the size of the committee to be elected is equal to one $(k=1)$, the CCR is equivalent to the Borda rule. However, it is easy to show that this is not necessarily the case when the target size of the committee is $k>1$. Recently, Kamwa and Merlin (2014) showed that with $m \geq 3$ competing candidates, the CCR is simply equivalent to the Plurality rule (defined later) for $k=m-1$. A natural question then arises, namely what about the cases where the target size of the committee is such that $1<k<m-1$ ? To the best of our knowledge, there is no analytical answer to this question; and we believe this could be due to the complexity of the CCR. In the first part of this paper we tackle this question by looking how often the outcome of the CCR may coincide with that of the following well-known multiwinner scoring rules: The $k$-Plurality rule, the $k$-Borda rule, the $k$-Negative Plurality rule and the Bloc rule according to various values of the pair $(k, m)$. More precisely, we compute the probabilities of agreement between the CCR and each of the above scoring rules for $m \in\{4,5,6\}$ and $1<k<m-1$ by considering various values for the number of voters (small number of voters as well as the case of large electorates). All our computations are done under the widely used hypothesis of the Impartial Anonymous Culture (defined later).

In the second part of this paper, we continue to bring out the differences between the $\mathrm{CCR}$ and the $k$-scoring rules that we focus on, but this time on the basis of the Condorcet principle. Indeed, what emerges from the debate between Dodgson (1884, 1876) and the Society for Proportional Representation is that when electing committees, the Condorcet principle cannot be totally ignored as it seems inconceivable to elect committees with dominated members or to elect dominated committees. A member of a committee is said to be dominated according to the Condorcet principle if there is an external candidate that is majority preferred to this member by more than half of the voters. In a recent paper,

\footnotetext{
${ }^{1}$ If the reader wants to know deeper on the complexity and the approximations of the CCR, we refer to the works of Betzler et al. (2013), Procaccia et al. (2008), Skowron et al. (2013a, 2015) and to the related literature.
} 
Diss and Doghmi (2016) have focused on the conditional probability for the $k$-Plurality rule, the $k$-Borda rule, the $k$-Negative Plurality rule and the Bloc rule to select the Condorcet committee à la Gehrlein when one exists: The Condorcet Committee Efficiency (CCE). The Condorcet committee à la Gehrlein is a subset of $k$ candidates such that each of its members defeats every outside candidate in pairwise majority comparisons (Gehrlein, 1985). It is clear that the CCE is simply the extension of the single-winner Condorcet efficiency of voting rules to multiwinner context as it is the conditional probability that a given voting rule elects the Condorcet winner when one exists. For further details on the Condorcet efficiency in the single-winner framework, the reader may refer to the works of Diss and Gehrlein (2015, 2012) and the recent books of Gehrlein and Lepelley (2017, 2011), among others. The second contribution of this paper is to compute the Condorcet committee efficiency of the CCR according to the same values of the pair $(k, m)$ that have been considered in Diss and Doghmi (2016), i.e., $m \in\{4,5,6\}$ and $1 \leq k \leq m-1$. Notice that we focus here on large electorates as in Diss and Doghmi (2016). Again, the probability model on which our results are based is the Impartial Anonymous Culture assumption.

The rest of the paper is structured as follows: Section 2 is devoted to basic notations and definitions. Section 3 presents our results on the probabilities of agreement between the $\mathrm{CCR}$ and the four multiwinner scoring rules listed above. Section 4 presents our results on the Condorcet committee efficiency. Section 5 concludes.

\section{Preliminaries}

Let $N=\{1, \ldots, n\}$ be a set of $n \geq 3$ voters and $A=\{a, b, c, \ldots\}$ the set of $m \geq 3$ candidates. We consider the framework in which each voter is assumed to have a linear order on the set of candidates from the most desirable candidate to the least desirable one. Notice that a linear order is a binary relation that is transitive, complete and antisymmetric. ${ }^{2}$ In addition, each voter is assumed to act according to her true preferences which clearly means that the setting of this paper does not support strategic voting. We denote by $\pi$ the preference profile of voters which identifies the specific linear ranking that each voter has on the candidates. In the sequel, we will simply write, $a b c \ldots$ to denote that candidate $a$ is ranked ahead candidate $b$ who is ranked ahead $c$ and so on. In this context, with $m$ candidates, there are $m$ ! possible strict rankings and a voting situation is defined by the vector $\tilde{n}=\left(n_{1}, \ldots, n_{t}, \ldots, n_{m !}\right)$ such that $\sum_{t=1}^{m !} n_{t}=n$. The notation $n_{t}$ refers to the number of voters endowed with each of the $m$ ! linear orders. For each pair of candidates $a, b \in A$, we denote by $n_{a b}$ the number of voters who rank candidate $a$ before candidate $b$. If $n_{a b}>n_{b a}$, we will say that candidate $a$ is majority preferred to candidate $b$ and we denote it by $a \mathbf{M} b$.

As our framework is that of multiwinner elections, we denote by $k(k<m)$ the number of candidates to be elected, i.e., the target size of the committee. In addition, we denote by

\footnotetext{
${ }^{2}$ The binary relation $R$ on $A$ is a subset of the cartesian product $A \times A$. We write $a R b$ if $(a, b) \in R$ and $\neg a R b$ if $(a, b) \notin R$. The binary relation $R$ on $A$ is transitive if $a R b$ and $b R c$ imply $a R c$ for all $a, b, c \in A$. $R$ is antisymmetric if $a R b$ and $b R a$ together imply $a=b . R$ is complete if, for all $a, b \in A$, we have $a R b$ or bRa.
} 
$\mathfrak{C}_{k}^{m}$ the set of all possible committees of size $k$ with $m$ candidates. A committee $C \in \mathfrak{C}_{k}^{m}$ is a Condorcet committee à la Gehrlein if each element in this committee beats each element not in it in terms of a pairwise majority comparison. Formally, this means that $\forall a \in C$ and $\forall b \in A \backslash C, a \mathbf{M} b$ is verified. As mentioned above, the Condorcet committee à la Gehrlein has been suggested in order to avoid committees with dominated member(s). Nonetheless, it does not always exist. That is the reason why other extensions of this concept have been suggested in the literature. We refer the reader to the works of Barberà and Coelho (2008), Kamwa (2017), Ratliff (2003) for more information on these notions. ${ }^{3}$

A scoring rule is a voting system that gives points to candidates in accordance with the position they occupy in voters' rankings. The total number of points received by a candidate defines her scores for the considered rule. In single-winner elections, the winner is the candidate with the highest score. Naturally, in multiwinner elections, the subset of winners or the elected committee is made by the candidates with the $k$ highest scores. The multiwinner rules considered in this paper are the following:

$k$-Plurality: This rule returns the $k$ candidates with the highest Plurality scores. The Plurality score of a candidate is the total number of voters who rank this candidate at the top of their rankings. Notice that the $k$-Plurality rule is also called Single Nontransferable Vote (e.g., Elkind et al., 2017).

$k$-Borda: It selects the candidates with the $k$ highest Borda scores. The Borda rule gives $m-j$ points to a candidate each time she is ranked $j$-th and the Borda score of a candidate is the sum of the points received.

$k$-Negative Plurality: Also called the $k$-Antiplurality rule, this rule returns the $k$ candidates with the lowest number of last place in the voters' rankings.

Bloc: This rule returns the $k$ candidates with the highest $k$-approval scores. The $k$-approval score of a candidate is equal to the number of voters who rank this candidate among their top $k$ ranked candidates. Notice that the Bloc rule is also called Limited Voting (Kamwa and Merlin, 2015) or Constant Scoring Rule (Gehrlein, 1985).

Chamberlin-Courant: Under this rule one fixes a scoring vector of length $m$, and each voter's score for a given committee is defined to be the score that she assigns to her most preferred candidate in that committee; the goal is then to find a committee that maximizes the joint scores of all voters. Notice that in line with Chamberlin and Courant (1983), we consider in this paper the most used vector of scores which is the

\footnotetext{
${ }^{3}$ Notice that the Condorcet committee à la Fishburn (Fishburn, 1981) has also been suggested in the literature in order to avoid dominated committees. In this framework, it is assumed that the voters have preferences over committees that satisfy certain conditions and Fishburn (1981) defines there a Condorcet committee as a committee that is preferred to every other committee by a majority of voters. Kamwa and Merlin (2018), Kaymak and Sanver (2003) have tried to bridge the two concepts through preference extensions. For their own purposes, Elkind et al. (2015) bridged these two concepts by introducing the notion of Condorcet winning set. A subset $B \subset A$ is a Condorcet winning set if for any candidate $a \in A \backslash B$, a majority of voters prefer some candidate in $B$ to $a$. Finally, we mention that other approaches are also taken into consideration by Ratliff (2006) and Brams et al. (2006) in order to compare sets of alternatives.
} 
one defined by Borda. Formally, let $r_{i x}$ be the rank of candidate $x$ in voter $i$ 's ranking and $w\left(r_{i x}\right)=m-r_{i x}$ the corresponding Borda weight. We denote by $N_{x}(C, \pi)$ the set of voters for which the representative in committee $C$ is candidate $x$ for profile $\pi$. In other words, for the corresponding profile $\pi, x$ is the most preferred candidate in the committee $C$ for all voters in $N_{x}(C, \pi)$. According to Chamberlin and Courant (1983), the CCR selects the committee which maximizes the representativeness value: ${ }^{4}$

$$
\alpha(C, \pi)=\sum_{x \in C} \sum_{i \in N_{x}(C, \pi)} w\left(r_{i x}\right)
$$

Recall that this paper only deals with the utilitarian approach of the CCR which is described in the definition above. Besides this approach, there are other ways of calculating the representativeness value. The reader interested in more details concerning this rule is referred to Betzler et al. (2013) who suggested the egalitarian variants of the CCR, some based on the Borda rule and others on the Approval rule. Notice that both the utilitarian and the egalitarian approaches are very hard to manage (Elkind et al., 2017). Lu and Boutilier (2011) have proposed some algorithms for approximating the CCR. For their own purposes, Potthoff and Brams (1998) showed that integer programming can be used in order to determine the winners under the CCR. Nevertheless, as pointed out by Procaccia et al. (2008), the integer programming does not overcome the complexity of the CCR.

In order to illustrate the different notions presented above, we provide the following two examples. On the one hand, Example 1 gives a profile in which the CCR leads to a committee that diverges from that chosen by the various $k$-scoring rules. On the other hand, Example 2 shows that this is not always the case since it provides a profile for which the CCR agree with every $k$-scoring rule studied in the paper.

Example 1. Consider the following profile $\pi_{1}$ on the set $A=\{a, b, c, d\}$ of $m=4$ candidates with $n=22$ voters:

\begin{tabular}{|c|c|c|c|c|c|}
\hline $\begin{array}{l}\text { voters: } \\
\text { rankings: }\end{array}$ & $\begin{array}{c}i=1, \ldots, 4 \\
a b c d\end{array}$ & $\begin{array}{c}i=5, \ldots, 8 \\
a d c b\end{array}$ & $\begin{array}{c}=9, \ldots, 12 \\
c b d a\end{array}$ & $\begin{array}{c}i=13, \ldots, 16 \\
b c d a\end{array}$ & $\begin{array}{c}i=17, \ldots, 22 \\
\text { dbca }\end{array}$ \\
\hline
\end{tabular}

The scores of the candidates under the Plurality rule, the Negative Plurality rule, the Borda rule, and the Bloc rule (with $k=2$ ) are as follows:

\footnotetext{
${ }^{4}$ It is important to notice that according to Chamberlin and Courant (1983), the CCR satisfies all the axioms used by Young (1974) in the characterization of the Borda rule namely the neutrality, the faithfulness, the consistency, and the cancellation axioms. All these axioms were adapted for committee selection function. A committee selection function is neutral if the committee selected does not depend upon the labelling of the committees. A committee selection function is faithful if, when applied to the preferences of a single individual, it selects the committees that best represent that individual. The consistency axiom works as follows: Consider two groups of voters that vote on the same set of candidates; if a committee belongs to the choice set of each group, consistency requires that it must also belong to the choice set when both groups are merged. A committee selection function has the cancellation property if, when the hypothesis holds, any individual' preference for committee $A$ over $B$ can be cancelled by some other individual's opposite preference with the result that all committees are considered equally representative.
} 


\begin{tabular}{|l|c|c|c|c|}
\cline { 2 - 5 } \multicolumn{1}{c|}{} & $a$ & $b$ & $c$ & $d$ \\
\hline Plurality & 8 & 4 & 4 & 6 \\
\hline Negative Plurality & 8 & 18 & 22 & 18 \\
\hline Borda & 24 & 40 & 34 & 34 \\
\hline Bloc & 8 & 18 & 8 & 10 \\
\hline
\end{tabular}

After all computations with $k=2$, the scores of the $6=\operatorname{Card}\left(\mathfrak{C}_{k=2}^{m=4}\right)$ possible committees under the CCR are:

$$
\begin{array}{ll}
\alpha\left(\{a, b\}, \pi_{1}\right)=56, & \alpha\left(\{a, c\}, \pi_{1}\right)=\alpha\left(\{a, d\}, \pi_{1}\right)=\alpha\left(\{c, d\}, \pi_{1}\right)=50, \\
\alpha\left(\{b, d\}, \pi_{1}\right)=54, & \alpha\left(\{b, c\}, \pi_{1}\right)=48 .
\end{array}
$$

In other words, if the target size of the elected committee is $k=2$, the CCR selects $\{a, b\}$ while the 2-Plurality rule chooses $\{a, d\}$; the committees $\{b, c\}$ and $\{c, d\}$ tie for the 2-Negative Plurality; the committees $\{b, c\}$ and $\{b, d\}$ tie for the 2-Borda rule; finally, the Bloc rule selects the committee $\{b, d\}$. As one can notice, the outcomes of the $k$-scoring rules differ from that of the CCR. Notice also that $\{b, c\}$ is the Condorcet committee à la Gehrlein (because bMa, bMd, cMa, and cMd) but it is not selected by the CCR.

Example 2. Consider the following profile $\pi_{2}$ on the set $A=\{a, b, c, d\}$ of $m=4$ candidates with $n=6$ voters:

\begin{tabular}{cccc}
\hline \hline voters: & $i=1, \ldots, 3$ & $i=4,5$ & $i=6$ \\
rankings: & abcd & bacd & dabc \\
\hline \hline
\end{tabular}

The scores of the candidates under the Plurality rule, the Negative Plurality rule, the Borda rule, and the Bloc rule (with $k=2$ ) are as follows:

\begin{tabular}{|l|c|c|c|c|}
\cline { 2 - 5 } \multicolumn{1}{c|}{} & $a$ & $b$ & $c$ & $d$ \\
\hline Plurality & 3 & 2 & 0 & 1 \\
\hline Negative Plurality & 6 & 6 & 5 & 1 \\
\hline Borda & 15 & 13 & 5 & 3 \\
\hline Bloc & 6 & 5 & 0 & 1 \\
\hline
\end{tabular}

After all computations with $k=2$, the scores of the committees under the CCR are:

$$
\begin{array}{ll}
\alpha\left(\{a, b\}, \pi_{2}\right)=17, \quad \alpha\left(\{a, d\}, \pi_{2}\right)=16, \quad \alpha\left(\{a, c\}, \pi_{2}\right)=\alpha\left(\{b, d\}, \pi_{2}\right)=15, \\
\alpha\left(\{b, c\}, \pi_{2}\right)=13, \quad \alpha\left(\{c, d\}, \pi_{2}\right)=8 .
\end{array}
$$

Thus, for $k=2$, the $C C R$ and all the $k$-scoring rules select the same committee $\{a, b\}$. Notice that this committee is also the Condorcet committee à la Gehrlein since a $\mathbf{M c}$, aMd, $b \boldsymbol{M} c$, and $b \boldsymbol{M} d$. 


\section{The agreement between Chamberlin-Courant rule and some multiwinner scoring rules}

As stated in Section 1, the CCR combines the Borda rule and the proportional representation; for $k=1$, the CCR is equivalent to the Borda rule and for $k=m-1$ the CCR is equivalent to the $k$-Plurality rule. Up to our knowledge, except these two values for the target size of the committee there are no other values of $k$ such that the CCR always coincides with a given $k$-scoring rule. For any pair $(k, m)$, our objective is then to find how often the outcome of the CCR coincides with that of the $k$-Plurality rule, the $k$-Borda rule, the $k$-Negative Plurality rule or the Bloc rule. For this purpose, we explore the cases for $m=4,5,6$. In order to find our probabilities, we assume that each voting situation is equally likely: This is called the Impartial Anonymous Culture (IAC). Formally, this model stipulates that all voting situations $\tilde{n}=\left(n_{1}, \ldots, n_{t}, \ldots, n_{m !}\right)$ for specified $n$ and $m$ are equiprobable with $n=\sum_{t=1}^{m !} n_{t}$. The integer $n_{t}$ being the number of voters endowed with the $t^{t h}$ corresponding linear order. Introduced by Gehrlein and Fishburn (1976) in social choice theory, the IAC assumption is one of the most used assumptions in the literature when computing the likelihood of voting events. For further details on the IAC and other assumptions, we refer the reader to the books of Gehrlein and Lepelley (2017, 2011).

\section{Methodology}

Before giving our results of this first contribution, we describe the methodology applied in order to calculate our probabilities. Under the IAC assumption, obtaining the probability of an electoral event is accomplished by the computation of two elements. The first one is the total number of voting situations $\tilde{n}=\left(n_{1}, \ldots, n_{t}, \ldots, n_{m !}\right)$; it is well-known that for $n$ voters and $m$ alternatives the total number of voting situations $\tilde{n}$ is given explicitly by the binomial coefficient $\left(\begin{array}{c}n+m !-1 \\ m !-1\end{array}\right)$. The second element to be calculated is the number of voting situations associated with the corresponding electoral event, which can be reduced to a finite system of linear constraints with rational coefficients.

As recently pointed out in the social choice literature, the most appropriate mathematical tools to deal with such problems are the Ehrhart polynomials. The background of this notion and its connection with the polytope theory can be found in Gehrlein and Lepelley (2017, 2011), Lepelley et al. (2008), and Wilson and Pritchard (2007). This technique has been widely used in numerous studies in order to evaluate the probability of electoral events in the case of three-candidate elections under the IAC assumption. For further information in this regard, we refer the reader to the recent studies of Courtin et al. (2015), Diss (2015), Diss et al. (2012), Gehrlein and Lepelley (2017, 2011), Gehrlein et al. (2017, 2016, 2015), Kamwa and Valognes (2017), Lepelley et al. (2018), and Smaoui et al. (2016). There are strong algorithms that enable to specify the Ehrhart polynomials for many problems in the case of three-candidate elections. ${ }^{5}$ As noticed in Lepelley et al. (2008), these algorithms do

\footnotetext{
${ }^{5}$ The most used general methods for computing Ehrhart polynomials are: Clauss's algorithm and Barvinok's algorithm. For more details, we recommend the report written by Verdoolaege et al. (2005).
} 
not allow to deal with four-candidate elections, where the total number of variables, i.e., possible linear orderings, is 24 . However, recent developments within the polytope theory allow us to obtain exact results for the case of $m=4$ and small number of voters $n \in[3,11]$. These results are obtained using the algorithms of Normaliz (Bruns et al., 2017) which is, to the best of our knowledge, the only program which is able to compute the number of voting situations in polytopes corresponding to elections with up to four candidates. The reader who is interested in a deeper understanding of the algorithms of Normaliz is refereed to Bruns et al. (2017) who describe several results obtained in four candidates elections. With this software, all computations in dimension 24 can be done but it needs relatively high memory when the number of voters increases. Consequently, computer simulations are used to evaluate our probabilities for $m=4$ and $n>11$ as well as for $m=5$ and $m=6$. We describe in the following the methodology applied in the simulations to estimate our probabilities under the IAC condition. Let us consider as an example the probability of agreement between the CCR and the $k$-Plurality rule for a specific pair $(k, m)$ and a given number of voters $n$. At the beginning, we randomly generate a voting situation of length $m$ ! with $n$ voters. In the second step, we check whether the conditions for which the CCR and the $k$-Plurality rule agree are fulfilled or not. These two steps are iterated 1,000,000 times to obtain the number of voting situations for which the CCR and the $k$-Plurality rule agree. $^{6}$ Finally, the probability of agreement is calculated as the quotient of the number previously obtained in the latest step over the total number of simulated voting situations, i.e., $1,000,000 .^{7}$ It is important to notice that another technique is used in this paper in order to obtain exact results when the number of candidates is $m=4$ and a number of voters tending to infinity. Indeed, it is well known that the calculations of the limiting probability under IAC condition are simply reduced to computation of volumes of convex polytopes. For this, our volumes are found with the use of the algorithm Convex which is a MAPLE package for convex geometry written by Franz (2016). This package works with the same general procedure that was implemented in Cervone et al. (2005) and recently used in other studies, e.g., Diss and Doghmi (2016), Diss and Gehrlein (2015, 2012), Gehrlein et al. (2015) and Moyouwou and Tchantcho (2017).

\section{Main results}

By investigating how often the CCR and one of the $k$-scoring rules agree, we compute three different probabilities that we deem relevant:

- Probability of agreement of type $1\left(P r_{1}\right)$ : When the CCR and the $k$-scoring rule both select the same one and only one committee. In this case, for a specific pair $(k, m)$, the symmetry of IAC with respect to candidates requires that the probability of only one case has to be found and then we multiply this result by the number of possible committees in order to find the total probability of agreement of type 1. For example, with $m=4$ and $k=2$, we first calculate the probability that the CCR and the $k$ scoring rule both select the committee $\{a, b\}$ and then we multiply this probability by

\footnotetext{
${ }^{6}$ Notice that we chose to run 1,000,000 iterations to generate our results with a very low margin of error.

${ }^{7}$ The MATLAB code of these simulations is available upon request from the authors.
} 
$6=\operatorname{Card}\left(\mathfrak{C}_{k=2}^{m=4}\right)$, to take into account the fact that the other possible committees can also be the winner for both rules instead of $\{a, b\}$.

- Probability of agreement of type $2\left(\mathrm{Pr}_{2}\right)$ : When the $k$-scoring rule selects only one committee and that this committee is included in the outcome set of the CCR that may contains more than one committee. It is obvious that $\mathrm{Pr}_{2}$ is equal to $\mathrm{Pr}_{1}$ plus the probability of the cases for which the $k$-scoring rule selects only one committee (e.g., $\{a, b\}$ ) and that the CCR chooses two or more committees including the one selected by the $k$-scoring rule (e.g., $\{a, b\},\{a, c\}$, and $\{b, c\}$ ). The symmetry of IAC with respect to candidates is also useful here to reduce the number of cases that we consider in order to find the total probability of agreement $\operatorname{Pr}_{2}$.

- Probability of agreement of type $3\left(P_{3}\right)$ : When a $k$-scoring rule selects one or more committees and that these committees are included in the outcome set of the CCR. In other words, the set of winning committees under the $k$-scoring rule is included in the one of the CCR. Again, it is obvious that $\mathrm{Pr}_{3}$ is equal to $\mathrm{Pr}_{2}$ plus the probability of the cases for which the $k$-scoring rule selects two of more committees (e.g., $\{a, b\}$ and $\{b, c\})$ and that the CCR chooses the committees selected by the $k$-scoring rule plus (or not) other possible committees with the same size (e.g., $\{a, b\},\{b, c\}$, and $\{a, c\}$ ). In this framework, although the symmetry of IAC with respect to candidates allows us to reduce the calculations, we notice that the number of cases still is incontestably high in particular for $m=5$ and $m=6$. For instance, with $m=6$ and $k=2$, in addition to the cases included in $\mathrm{Pr}_{2}$, we need to conduct the calculations for the probability of the situations for which the $k$-scoring rule selects $2,3,4,5,6,10$, or 15 committees $^{8}$ of size $k=2$ with the requirement that the set of its winning committees is included in the one of the CCR. Let us give an example. Suppose that the $k$-scoring rule selects two committees which belong to the set of winning committees of the CCR. This case corresponds to all voting situations under which the $k$-scoring rule ranks exactly one candidate (suppose $a$ ) in the first position followed by two other candidates (suppose $b$ and $c$ ) who tied in the second place followed by the other candidates in the last position with or without ties. In other words, the possible winning committees under the $k$-scoring rule are $\{a, b\}$ and $\{a, c\}$ which should be selected by the CCR in addition (or not) to other possible committees of the same size. Evidently, using the symmetry of IAC with respect to candidates, the probability of this situation has to be multiplied by $60=\left(\begin{array}{l}6 \\ 1\end{array}\right) \times\left(\begin{array}{l}5 \\ 2\end{array}\right)$ to take into account the fact that the other candidates can also be ranked in the first and second places instead of $a, b$, and $c$.

Obviously, the probability $\operatorname{Pr}_{1}$ analyses the cases where the rules are decisive (also called resolute); the probability $\mathrm{Pr}_{2}$ assumes that only the $k$-scoring rule is decisive while the CCR is not and $\mathrm{Pr}_{3}$ supposes that both the CCR and the $k$-scoring rule are not decisive. We deem relevant to distinguish the cases where the rules are decisive from those they are not

\footnotetext{
${ }^{8}$ These values correspond to the possible cardinality of the set of winning committees among $15=$ $\operatorname{Card}\left(\mathfrak{C}_{k=2}^{m=6}\right)$ possible committees when the social (collective) ranking on candidates is accomplished under the $k$-scoring rule. The case of 1 winning committee is considered in the probability $\operatorname{Pr}_{2}$ and the cases 7,8 , $9,11,12,13$, and 14 are obviously not possible.
} 
because breaking ties may lead to more complex problems: the choice of the tie-breaking rule is not entirely neutral because it can be used for strategic manipulation purposes as recently pointed out by Aziz al. (2013), Mattei et al. (2014), Obraztsova et al. (2011).

Let us admit that apart from $\operatorname{Pr}_{1}, \mathrm{Pr}_{2}$ and $P r_{3}$, other types of probabilities can be considered. As one can notice, $\operatorname{Pr}_{1}, \operatorname{Pr}_{2}$ and $P r_{3}$ address the agreement by only assuming an overlap of the outcome sets that focuses on the committees as a whole. One can go further and imagine the agreement in terms of members; in other words, one would determine the probability that a candidate elected with a $k$-scoring rule will also be chosen by the CCR with (or without) the same value of $k$. Exploring the agreement in this way is interesting but it may give rise to the study of some tricky situations as the following one: For $k=2$ assume that a $k$-scoring rule selects the two committees $\{a, b\}$ and $\{a, c\}$ while the CCR selects the committee $\{b, c\}$. In such a case, we get at the same time an agreement on candidates $b$ and $c$ and also a disagreement on candidate $a$. Given this particular case, assume that there is a bill that advocates the adoption of the CCR instead of the $k$-scoring rule and that the likelihood of this particular case is not negligible: If candidate $a$ knows that she has great chances of being appointed no matter what is the elected committee under the $k$-scoring rule, she will strongly militate against the constitutional change (the adoption of the CCR) and she will promote the status quo (the $k$-scoring rule). So, analyzing the agreement in terms of members can also be used to explain the (dis)incentives for constitutional changes.

The results of our computations are provided in Tables 6 to 11 in the Appendix; Figures 1 to 3 provide a global overview that allows us to glimpse the possible comparisons between the rules.

The probability $P r_{1}$ tends to increase with the size of the electorate given $m$ and $k$. For instance, with $(k, m)=(2,4)$, the probability of agreement grows from $38.05 \%$ with four voters to $72.23 \%$ when the electorate tends to infinity for the $k$-Plurality rule; for the $k$-Borda rule, it grows form $32.55 \%$ to $57.38 \%$; for the Bloc rule, it grows from $23.08 \%$ to $45.37 \%$; and for the $k$-Negative Plurality rule, it grows from $13.85 \%$ to $31.93 \%$. In term of agreement with the CCR, the $k$-Plurality rule dominates the other $k$-scoring rules in almost all cases. More precisely, for $(k, m) \in\{(2,4),(3,5),(3,6),(4,6)\}$, the $k$-Plurality rule clearly dominates the other $k$-scoring rules; it is followed by the $k$-Borda rule, then the Bloc rule and the $k$-Negative Plurality rule performs the worst. For $(2,5)$, the $k$-Plurality rule and the $k$-Borda rule clearly dominates the Bloc rule which dominates the $k$-Negative Plurality rule; in this case, as the size of the electorate increases, the dominance between the $k$-Plurality rule and the $k$-Borda rule alternates. For $(2,6)$, the $k$-Negative Plurality rule performs the worst while the $k$-Borda rule dominates the $k$-Plurality rule for $n \geq 8$; in this case, the Bloc rule dominates the $k$-Plurality rule for $n \geq 50$. In sum, with respect to the probability of agreement of type 1 , the $k$-Plurality rule tends to dominate the other $k$-scoring rules while the $k$-Negative Plurality rule performs the worst.

Concerning $\operatorname{Pr}_{2}$, we get similar conclusions as with $P r_{1}$. Except for the pairs $(3,5)$ and $(3,6)$, in most of the cases, the $k$-Plurality rule tends to more agree with the CCR, it is followed by the $k$-Borda rule, then the Bloc rule and the $k$-Negative Plurality rule makes it worst. It is important to notice that, for a given $k$-scoring rule and a pair $(k, m)$, as the size of 
the electorate tends to be large the result $P r_{2}=P r_{1}$ holds. Why? Recall that $P r_{2}$ is equal to $\operatorname{Pr}_{1}$ plus the probability of the cases for which the $k$-scoring rule selects only one committee which must be included in the set of the winning committees of the CCR containing at least two committees. To obtain $\mathrm{Pr}_{1}$, our polytopes are only described by strict inequalities while under $\mathrm{Pr}_{2}$ we need weak inequalities. We know that when $n$ increases more and more, these weak inequalities fall to make room for strict inequalities and we recover $\operatorname{Pr}_{1}{ }^{9}$

Recall that $\mathrm{Pr}_{3}$ is equal to $\mathrm{Pr}_{2}$ plus the probability of the cases for which the $k$-scoring rule selects two of more committees while the CCR chooses the committees selected by the $k$-scoring rule plus (or not) other possible committees of the same size. We get similar conclusions as with $\mathrm{Pr}_{2}$. More precisely, when both the CCR and a $k$-scoring rule are both non decisive, the CCR is more likely to agree with the $k$-Plurality rule than with the other $k$ scoring rules. This probability is definitely weaker with the $k$-Negative Plurality rule. Once again, for a given $k$-scoring rule and the pairs $(k, m)$, we get $P r_{3}=\mathrm{Pr}_{2}$ for large electorates. The same argument used to explain why $P r_{1}=P r_{2}$ also stands here; more, the probability that we add to $P r_{2}$ in order to get $P r_{3}$ goes very quickly to 0 and often it is equal to 0.0000 with 1000 or 1001 voters.

To summarize, under the probabilities $P r_{1}$ and $P r_{3}$, except for the pairs $(2,5)$ and $(2,6)$, there is a clear-cut that no matter the size of the electorate, the CCR agrees most of time with the $k$-Plurality rule than with the other $k$-scoring rules. Under $\operatorname{Pr}_{2}$, as the size of the electorate varies, the agreement alternates between the $k$-Plurality rule and the $k$-Borda rule. From the above, we note that the CCR leads in most of the cases to a committee made by the candidates with the $k$ greatest Plurality scores.

\footnotetext{
${ }^{9}$ As noticed above, the calculations of the limiting probability under IAC condition are simply reduced to computations of volumes of convex polytopes. Discounting ties has no impact because the volume of a region is the same regardless of whether the region includes its bounding hyperplanes or not, and it is exactly the points lying on these bounding hyperplanes that correspond to the ties (included in the voting situations of $\mathrm{Pr}_{2}$ and not in $\mathrm{Pr}_{1}$ ). For more details, we refer the reader to footnote 3 in Cervone et al. (2005).
} 
$\mathbf{m}=4 ; k=2$

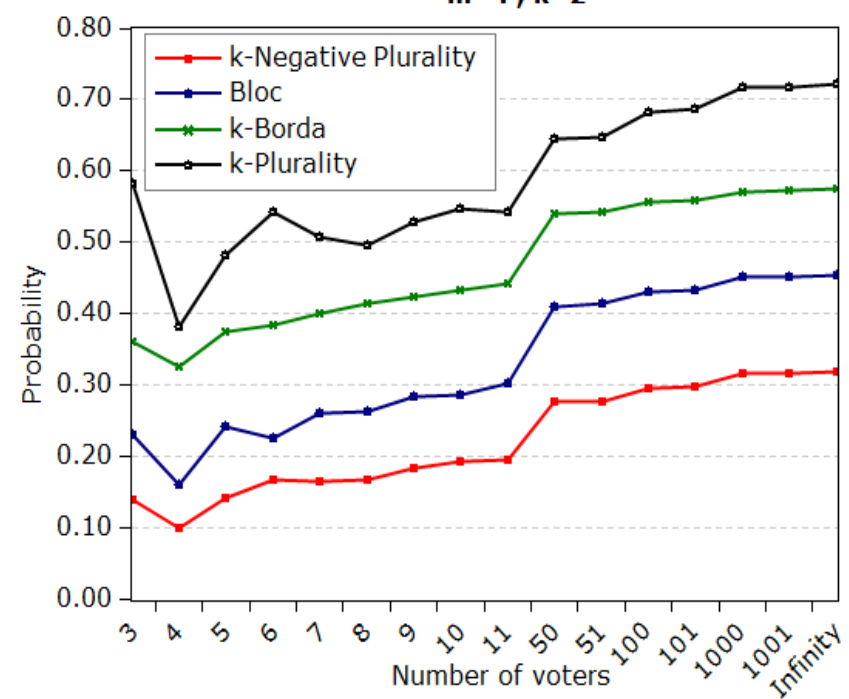

$\mathbf{m}=5 ; \mathbf{k}=3$

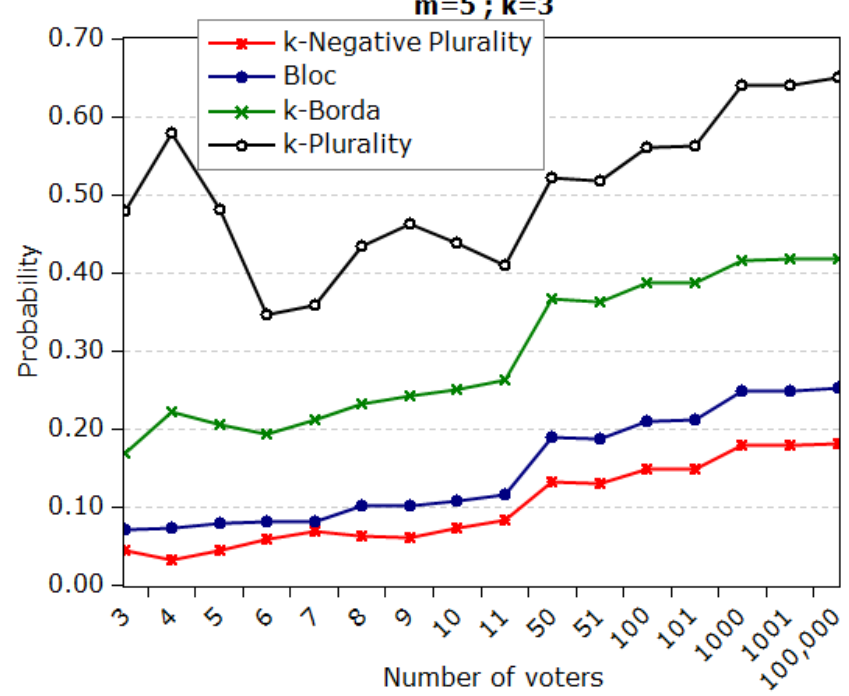

$m=6 ; k=3$

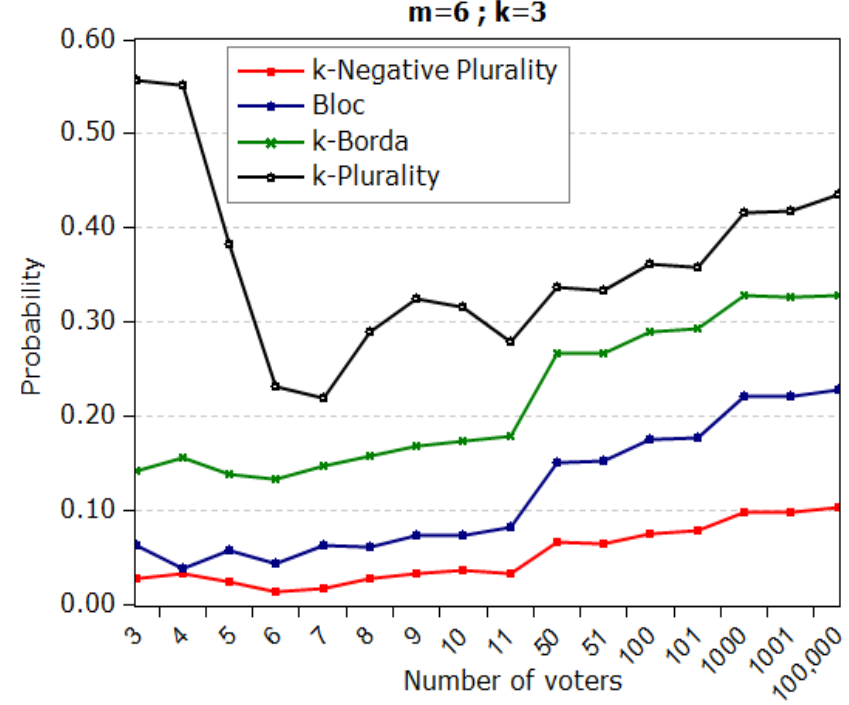

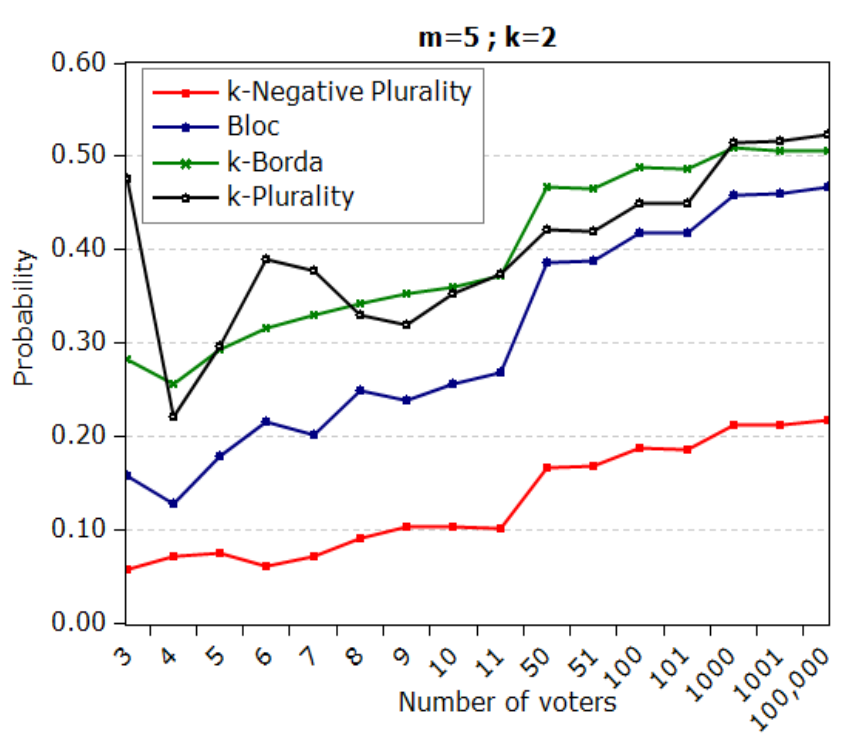

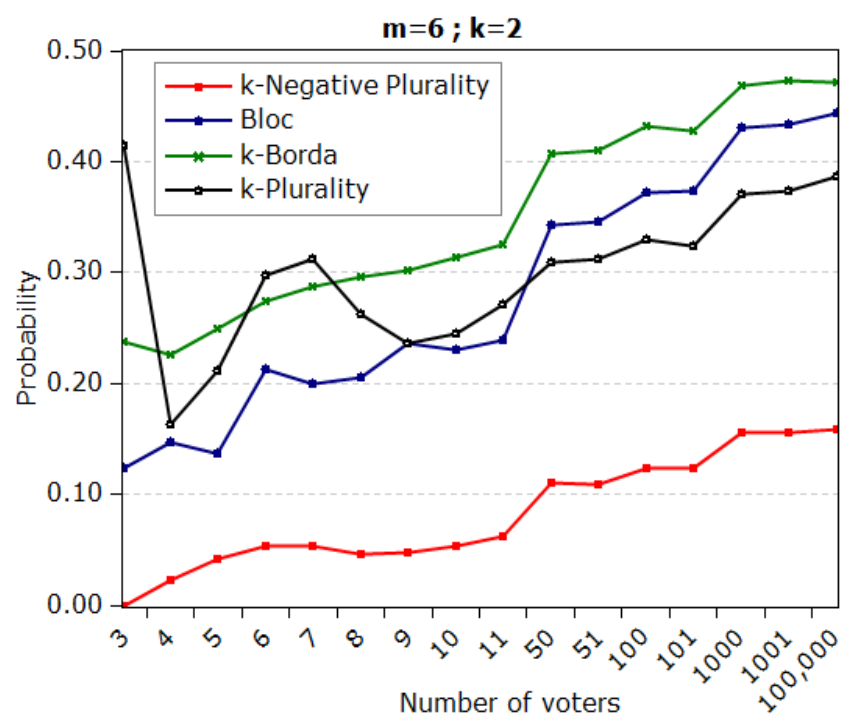

$\mathbf{m}=6 ; \mathbf{k}=4$

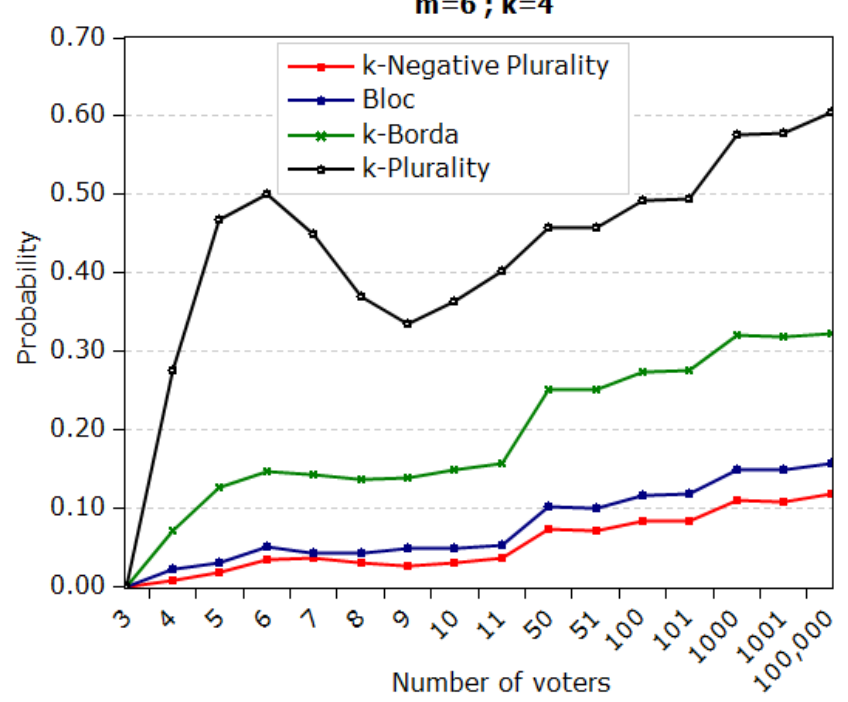

Figure 1: The probability of type 1 for the agreement between Chamberlin-Courant and other multiwinner scoring rules 
$\mathbf{m}=4 ; k=2$

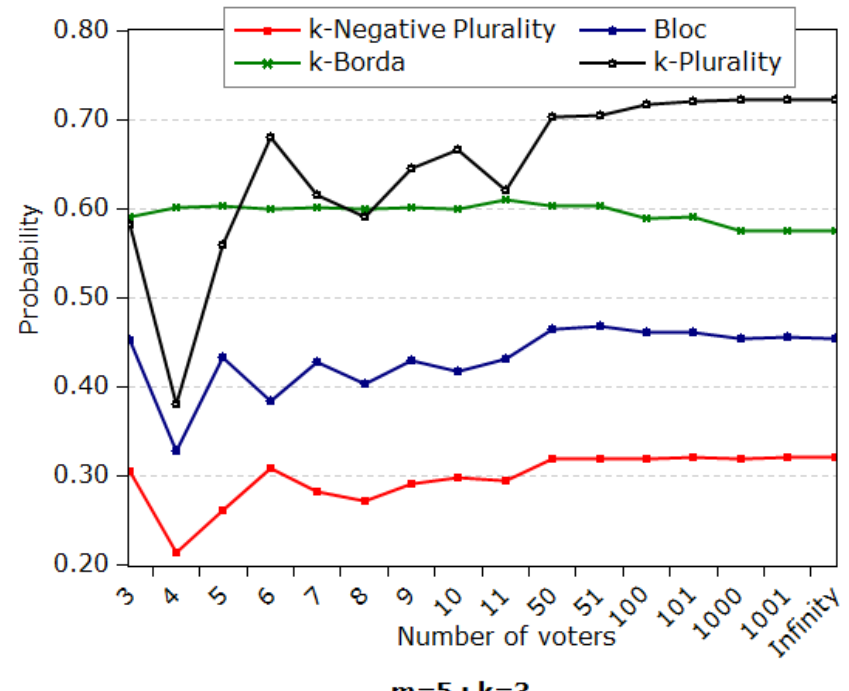

$\mathbf{m}=5 ; k=3$
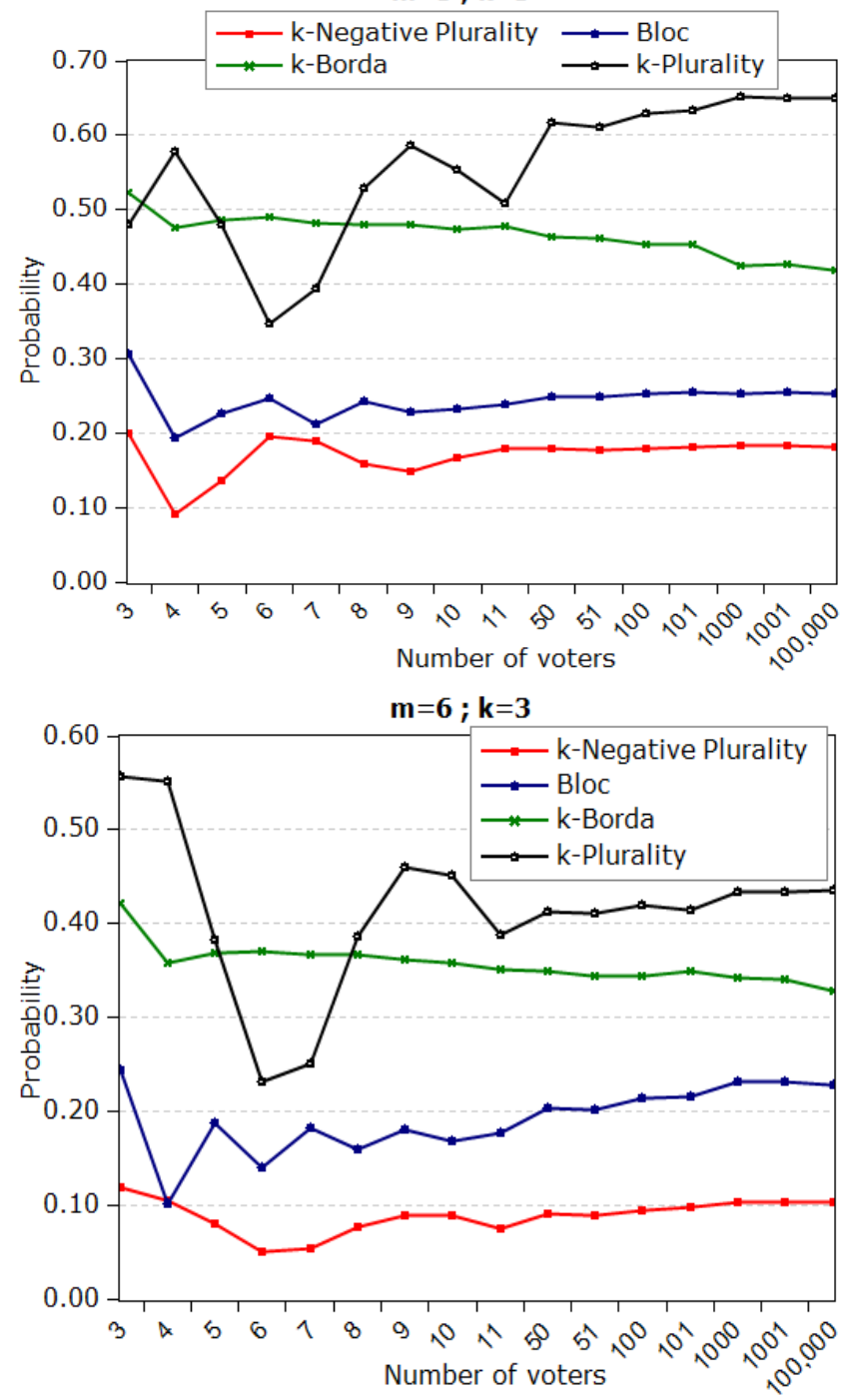

$\mathbf{m}=5 ; \mathbf{k}=2$

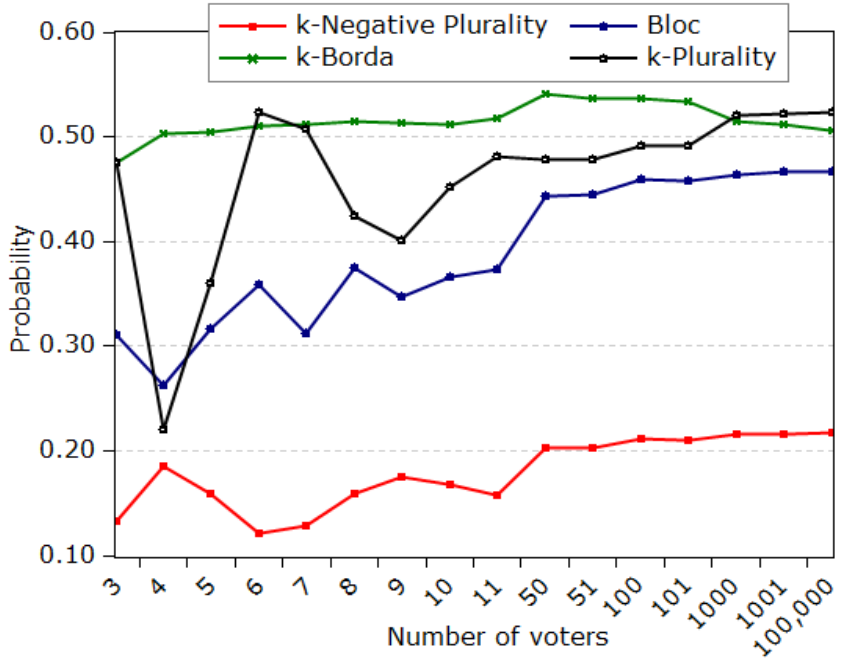

$\mathrm{m}=6 ; \mathrm{k}=2$

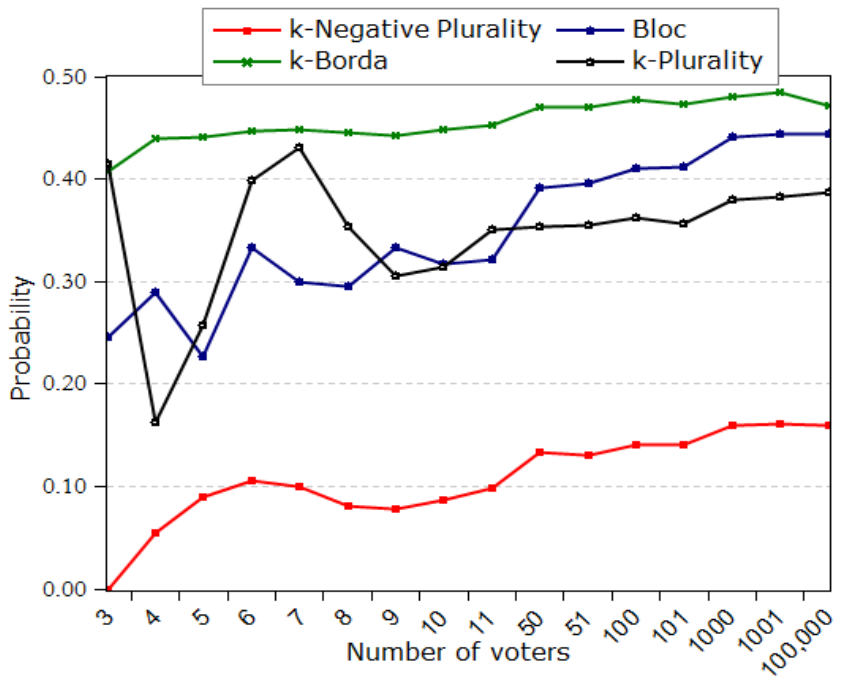

$m=6 ; k=4$

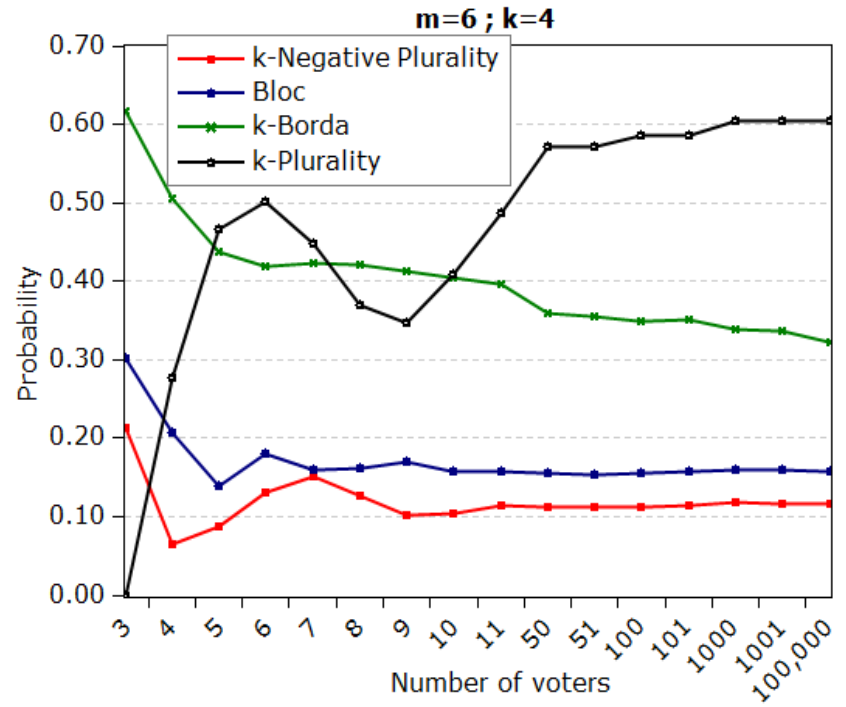

Figure 2: The probability of type 2 for the agreement between Chamberlin-Courant and other multiwinner scoring rules 
$m=4 ; k=2$

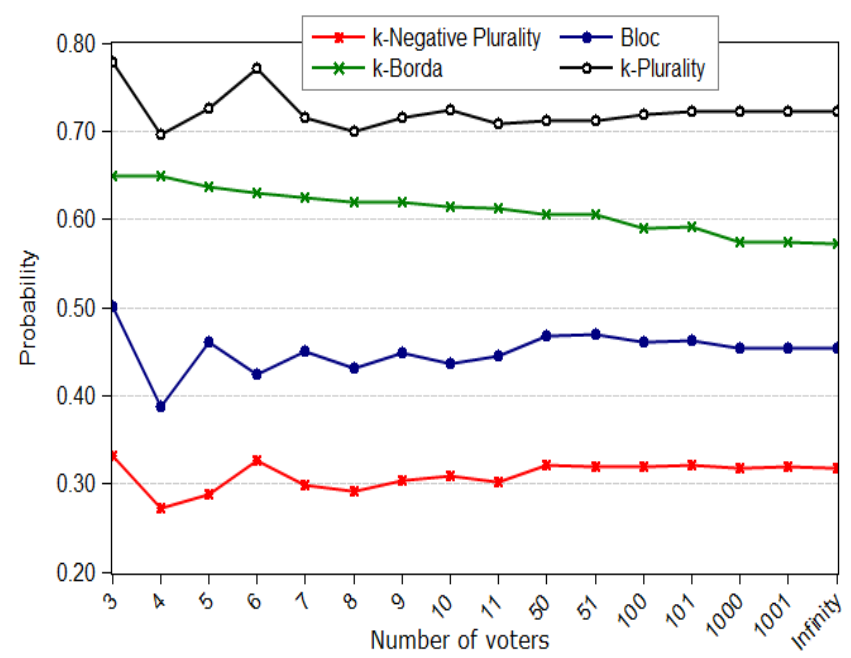

$m=5 ; k=3$

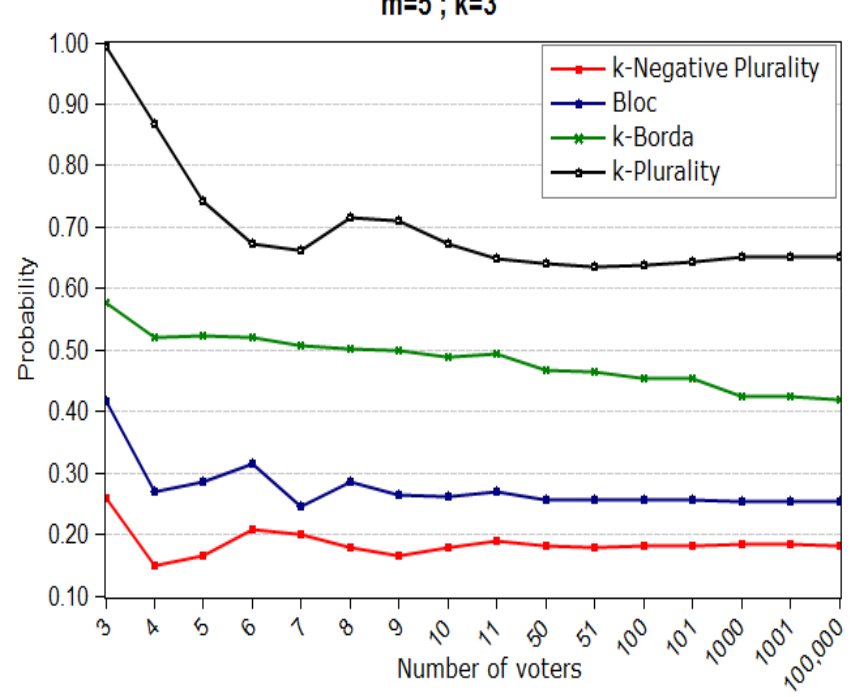

$m=6 ; k=3$

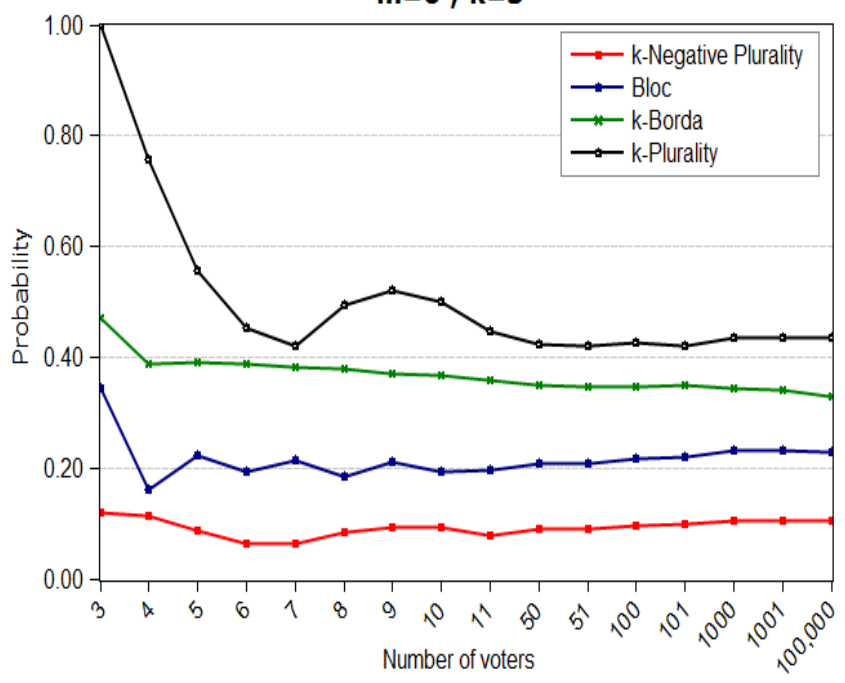

$m=5 ; k=2$

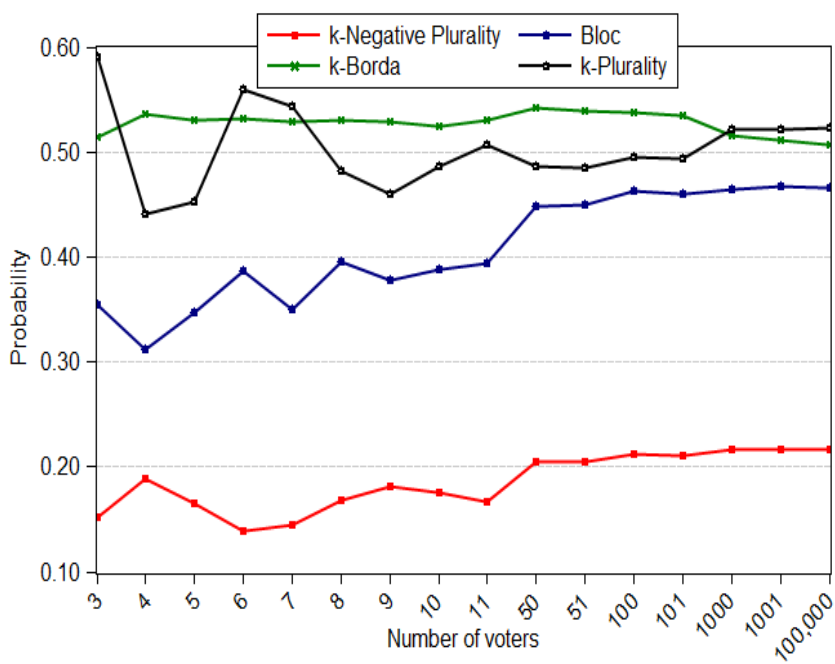

$\mathrm{m}=6$; $\mathrm{k}=2$

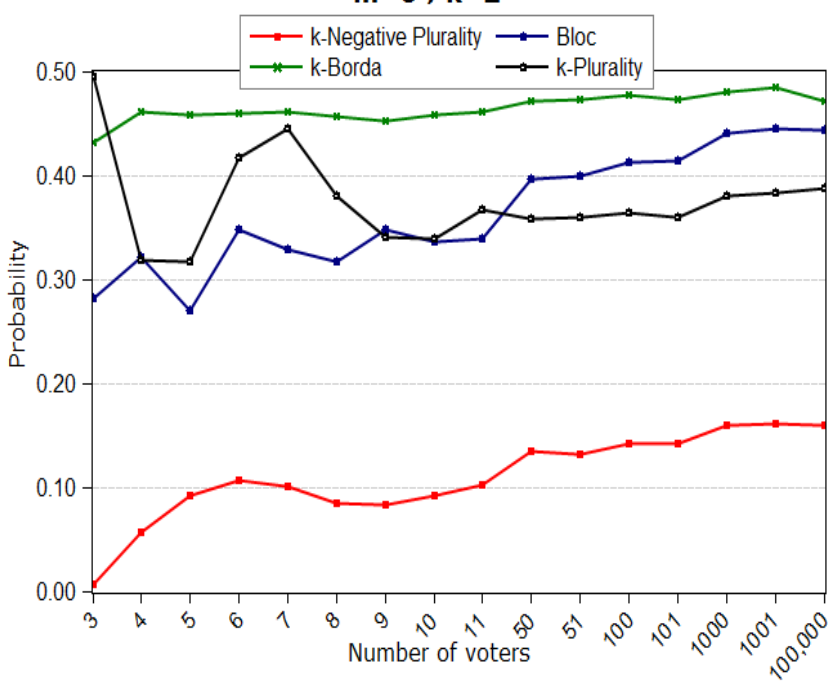

$m=6$; $k=4$

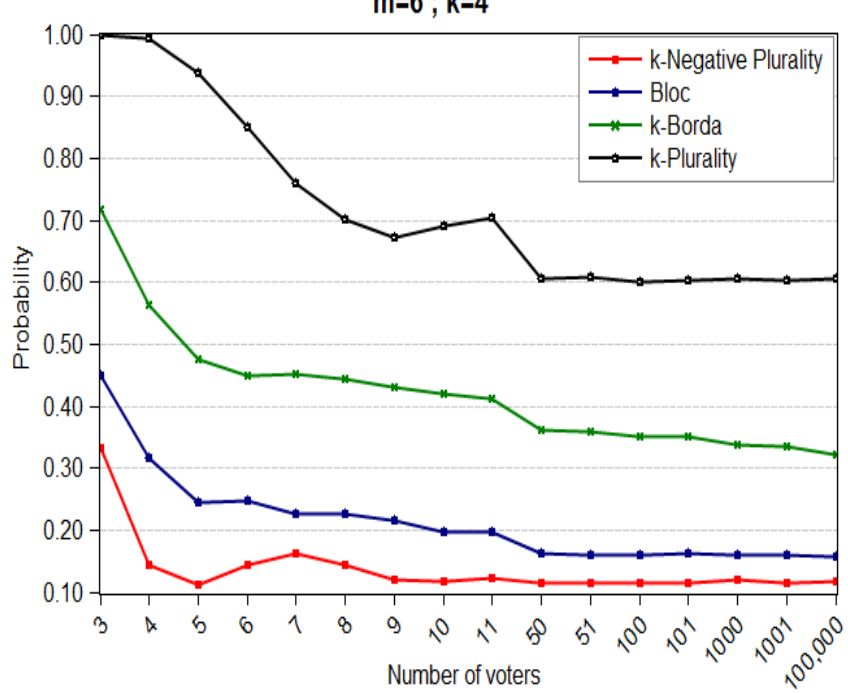

Figure 3: The probability of type 3 for the agreement between Chamberlin-Courant and other multiwinner scoring rules 


\section{The Condorcet committee efficiency of the Chamberlin- Courant rule}

We continue here the study of the differences between the CCR and the $k$-scoring rules that we focus on in this paper by evaluating the probability of the CCR to select the Condorcet committee $\grave{a}$ la Gehrlein when it exists. We also compare our results with the ones obtained in Diss and Doghmi (2016) for the other multiwinner rules.

\section{Methodology}

In order to evaluate the Condorcet Committee Efficiency (CEE) for the case of $m=4$ candidates, we use here the same method of volumes that has been implemented in the previous section for calculating the probability of agreement between the CCR and the other multiwinner scoring rules. Again, it turns out to be impossible to obtain results in an acceptable time for $m=5$ and $m=6$. Consequently, to circumvent the impossibility of exact results, we perform a computer simulation to estimate these probabilities using the Monte-Carlo simulation methodology in the spirit of the IAC assumption. As in the previous section, we take into consideration $n=100,000$ voters and we run 1,000,000 elections in order to generate our results with a very low margin of error. It is important to precise that the results in Diss and Doghmi (2016) are obtained with the same number of voters but with the generation of 100,000 iterations instead of 1,000,000. The difference in the number of the generated elections in this paper and the one by Diss and Doghmi (2016) has no impact on the results since in both cases our results guarantee a very low margin of error.

\section{Main results}

We denote by $C C E_{I A C \infty}^{k-P}(k, m), C C E_{I A C \infty}^{k-N P}(k, m), C C E_{I A C \infty}^{k-B}(k, m), C C E_{I A C \infty}^{B}(k, m)$ and $C C E_{I A C \infty}^{C C R}(k, m)$, the CCE of the $k$-Plurality rule, the $k$-Negative Plurality rule, the $k$ Borda rule, the Bloc rule, and the CCR, respectively. Tables 1 to 5 provide the CCE of the five multiwinner scoring rules that we consider in this paper. Figure 4 gives a global overview of the results. Notice that Tables 2 to 5 are drawn from Diss and Doghmi (2016). In other words, our findings in this section come down in Table 1 which provides the CCE of the CCR for various values of $m$ and $k$.

First, our results show that the CCE of the CCR tends to decrease when going from $k=1$ to $k<m-1$ and then it increases at $k=m-1$. For instance, for $m=5$, the CCE of the CCR varies from 0.8537 for $k=1$ to 0.4378 for $k=3$ and then it reaches 0.5106 for $k=4$; for $m=6$ the CCE of the CCR varies from 0.8458 for $k=1$ to 0.3574 for $k=4$ and then it reaches 0.4731 for $k=5$. As expected, we get the equivalence between the CCR and respectively the $k$-Borda rule $(k=1)$ and the $k$-Plurality rule $(k=m-1)$. 
Table 1: $C C E_{I A C_{\infty}}^{C C R}(k, m) \quad$ Table 2: $C C E_{I A C_{\infty}}^{k-P}(k, m) \quad$ Table 3: $C C E_{I A C_{\infty}}^{k-N P}(k, m)$

\begin{tabular}{|c|c|c|c|c|c|c|c|c|c|c|c|}
\hline $\begin{array}{c}m \rightarrow \\
k \downarrow\end{array}$ & 4 & 5 & 6 & $\begin{array}{c}m \rightarrow \\
k \downarrow\end{array}$ & 4 & 5 & 6 & $\begin{array}{c}m \rightarrow \\
k \downarrow\end{array}$ & 4 & 5 & 6 \\
\hline 1 & 0.8706 & 0.8537 & 0.8458 & 1 & 0.7426 & 0.6143 & 0.5207 & 1 & 0.5516 & 0.5104 & 0.4696 \\
\hline 2 & 0.5642 & 0.5398 & 0.5182 & 2 & 0.5427 & 0.4476 & 0.3638 & 2 & 0.5427 & 0.4267 & 0.3529 \\
\hline 3 & 0.5516 & 0.4378 & 0.3757 & 3 & 0.5516 & 0.4199 & 0.3380 & 3 & 0.7426 & 0.4521 & 0.3211 \\
\hline 4 & -- & 0.5106 & 0.3574 & 4 & -- & 0.5100 & 0.3322 & 4 & -- & 0.6140 & 0.3642 \\
\hline 5 & -- & -- & 0.4731 & 5 & -- & -- & 0.4701 & 5 & -- & -- & 0.5183 \\
\hline
\end{tabular}

Table 4: $C C E_{I A C_{\infty}}^{k-B}(k, m)$

\begin{tabular}{|c|ccc}
\hline $\begin{array}{c}m \rightarrow \\
k \downarrow\end{array}$ & 4 & 5 & 6 \\
\hline 1 & 0.8706 & 0.8580 & 0.8457 \\
2 & 0.8598 & 0.8286 & 0.8234 \\
3 & 0.8706 & 0.8348 & 0.8172 \\
4 & -- & 0.8587 & 0.8372 \\
5 & -- & -- & 0.8477 \\
\hline
\end{tabular}

Table 5: $C C E_{I A C_{\infty}}^{B}(k, m)$

\begin{tabular}{|c|ccc}
\hline $\begin{array}{c}m \rightarrow \\
k \downarrow\end{array}$ & 4 & 5 & 6 \\
\hline 1 & 0.7426 & 0.6143 & 0.5207 \\
2 & 0.7468 & 0.6210 & 0.5386 \\
3 & 0.7426 & 0.6337 & 0.5715 \\
4 & -- & 0.6140 & 0.5330 \\
5 & -- & -- & 0.5183 \\
\hline
\end{tabular}

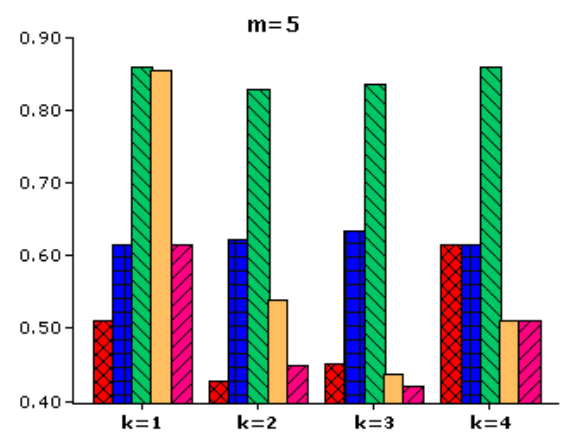

$\mathbf{m}=6$

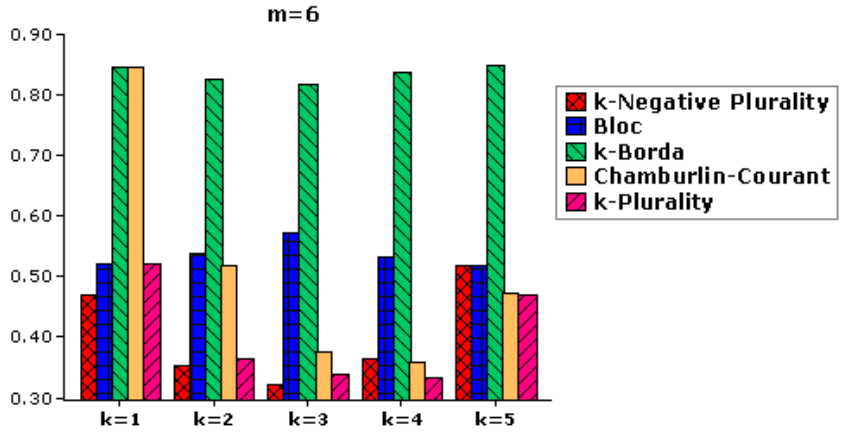

Figure 4: The Condorcet committee efficiency of Chamberlin-Courant and other multiwinner scoring rules. 
Second, as already noticed by Diss and Doghmi (2016), the $k$-Borda rule tends to perform better than the other $k$-scoring rules; it is followed by the Bloc rule. The $k$-Plurality rule performs the worst. Bringing our results to those of Diss and Doghmi (2016), it comes out that the CCR does not perform better than the $k$-Borda rule and the Bloc rule (except for $k=1$ ); it performs however better than the $k$-Plurality rule. We notice that for $k \leq\left\lfloor\frac{m}{2}\right\rfloor$, the CCR performs better than the $k$-Negative Plurality rule and for $k>\left\lfloor\frac{m}{2}\right\rfloor$ we get the reverse, where $L\rfloor$ stands for the integer part of the number. To summarize, though the CCR is fitted to produce diverse (proportional) committees, our findings on the CCE tell us that this rule can lead to committees with dominated members most of the time than the $k$-Borda rule and the Bloc rule.

\section{Concluding remarks}

Despite the fact that the Chamberlin-Courant rule is appropriate to ensure proportional representation, computing the outcomes can be computationally intractable (see for instance Betzler et al., 2013, Procaccia et al., 2008, Skowron et al., 2013a, 2015). As this drawback may compromise the use of this rule in real elections, the aim of this paper was to determine how often the outcome of the CCR may coincide with that of the following well-known rules: The $k$-Plurality rule, the $k$-Borda rule, the $k$-Negative Plurality rule and the Bloc rule. We focused on voting situations with $m=4,5,6$ candidates and committees of size $1<k<m-1$. We found that in most of the cases, the $k$-Plurality rule tends to more agree with the CCR, it is followed by the $k$-Borda, then the Bloc rule and the $k$-Negative Plurality makes it worst. Results for $m>6$ would have allowed to draw more accurate results. Obtaining them comes up against the limits of probability calculations under IAC assumption even with computer simulations.

We have also drawn a comparison between the CCR and the above four multi-winner scoring rules on the basis of their propensity to select the Condorcet committee à la Gehrlein. when it does exist: The Condorcet Committee Efficiency. Electing the Condorcet committee à la Gehrlein when it exists guarantees a committee with undominated members. Our results told us that the CCR does not perform better than the $k$-Borda rule and the Bloc rule; it performs better than the $k$-Plurality rule. Moreover, the CCR performs better than the $k$-Negative Plurality rule for a size of the committee smaller than half of the number of the competing candidates.

\section{Appendix}

Recall that Tables 6 to 11 provide the probabilities $P r_{1}, P r_{2}$, and $P r_{3}$ of agreement between the CCR and each of the multiwinner scoring rules that we consider in this paper with various values of $m$ and $k$, the number of candidates and the size of the target committee, respectively. 
Table 6: The probability of agreement between Chamberlin-Courant and other multiwinner scoring rules, $m=4$ and $k=2$

\begin{tabular}{|c|ccc|ccc|cccc|ccc|}
\hline \multirow{2}{*}{$n$} & \multicolumn{2}{|c|}{$\begin{array}{c}\text { Chamberlin-Courant } \\
\text { and } k \text {-Plurality }\end{array}$} & \multicolumn{2}{c|}{$\begin{array}{c}\text { Chamberlin-Courant } \\
\text { and } k \text {-Borda }\end{array}$} & \multicolumn{2}{c|}{$\begin{array}{c}\text { Chamberlin-Courant } \\
\text { and Bloc }\end{array}$} & \multicolumn{2}{c|}{$\begin{array}{c}\text { Chamberlin-Courant and } \\
k \text {-Negative Plurality }\end{array}$} \\
\cline { 2 - 14 } & $P r_{1}$ & $P r_{2}$ & $P r_{3}$ & $P r_{1}$ & $P r_{2}$ & $P r_{3}$ & $P r_{1}$ & $P r_{2}$ & $P r_{3}$ & $P r_{1}$ & $P r_{2}$ & $P r_{3}$ \\
\hline 3 & 0.5815 & 0.5815 & 0.7785 & 0.3600 & 0.5908 & 0.6492 & 0.2308 & 0.4523 & 0.5015 & 0.1385 & 0.3046 & 0.3323 \\
4 & 0.3805 & 0.3805 & 0.6964 & 0.3255 & 0.6003 & 0.6496 & 0.1590 & 0.3265 & 0.3874 & 0.0991 & 0.2126 & 0.2728 \\
5 & 0.4808 & 0.5590 & 0.7265 & 0.3734 & 0.6020 & 0.6366 & 0.2415 & 0.4330 & 0.4608 & 0.1404 & 0.2598 & 0.2882 \\
6 & 0.5426 & 0.6802 & 0.7720 & 0.3843 & 0.5996 & 0.6301 & 0.2242 & 0.3841 & 0.4246 & 0.1665 & 0.3087 & 0.3259 \\
7 & 0.5080 & 0.6143 & 0.7151 & 0.3998 & 0.6017 & 0.6250 & 0.2609 & 0.4264 & 0.4498 & 0.1638 & 0.2810 & 0.2988 \\
8 & 0.4952 & 0.5904 & 0.6992 & 0.4140 & 0.5988 & 0.6197 & 0.2614 & 0.4031 & 0.4305 & 0.1669 & 0.2707 & 0.2924 \\
9 & 0.5290 & 0.6440 & 0.7150 & 0.4233 & 0.6011 & 0.6187 & 0.2825 & 0.4282 & 0.4477 & 0.1825 & 0.2899 & 0.3039 \\
10 & 0.5474 & 0.6658 & 0.7240 & 0.4326 & 0.5984 & 0.6140 & 0.2846 & 0.4158 & 0.4362 & 0.1934 & 0.2968 & 0.3082 \\
11 & 0.5434 & 0.6513 & 0.7092 & 0.4416 & 0.5989 & 0.6125 & 0.3009 & 0.4299 & 0.4458 & 0.1951 & 0.2909 & 0.3025 \\
50 & 0.6460 & 0.7032 & 0.7117 & 0.5405 & 0.6033 & 0.6053 & 0.4104 & 0.4647 & 0.4675 & 0.2760 & 0.3191 & 0.3208 \\
51 & 0.6483 & 0.7046 & 0.7125 & 0.5428 & 0.6034 & 0.6052 & 0.4135 & 0.4670 & 0.4693 & 0.2762 & 0.3183 & 0.3201 \\
100 & 0.6825 & 0.7160 & 0.7184 & 0.5558 & 0.5887 & 0.5894 & 0.4308 & 0.4601 & 0.4608 & 0.2954 & 0.3186 & 0.3192 \\
101 & 0.6863 & 0.7194 & 0.7216 & 0.5577 & 0.5909 & 0.5913 & 0.4323 & 0.4613 & 0.4621 & 0.2972 & 0.3203 & 0.3207 \\
1000 & 0.7183 & 0.7220 & 0.7220 & 0.5709 & 0.5744 & 0.5744 & 0.4503 & 0.4534 & 0.4534 & 0.3152 & 0.3176 & 0.3176 \\
1001 & 0.7180 & 0.7215 & 0.7216 & 0.5716 & 0.5750 & 0.5750 & 0.4513 & 0.4544 & 0.4544 & 0.3170 & 0.3193 & 0.3193 \\
$\infty$ & 0.7223 & 0.7223 & 0.7223 & 0.5732 & 0.5732 & 0.5732 & 0.4537 & 0.4537 & 0.4537 & 0.3173 & 0.3173 & 0.3173 \\
\hline
\end{tabular}

Table 7: The probability of agreement between Chamberlin-Courant and other multiwinner scoring rules, $m=5$ and $k=2$

\begin{tabular}{|c|c|c|c|c|c|c|c|c|c|c|c|c|}
\hline \multirow[t]{2}{*}{$n$} & \multicolumn{3}{|c|}{$\begin{array}{c}\text { Chamberlin-Courant } \\
\text { and } k \text {-Plurality }\end{array}$} & \multicolumn{3}{|c|}{$\begin{array}{l}\text { Chamberlin-Courant } \\
\text { and } k \text {-Borda }\end{array}$} & \multicolumn{3}{|c|}{$\begin{array}{c}\text { Chamberlin-Courant } \\
\text { and Bloc }\end{array}$} & \multicolumn{3}{|c|}{$\begin{array}{c}\text { Chamberlin-Courant and } \\
k \text {-Negative Plurality }\end{array}$} \\
\hline & $P r_{1}$ & $\mathrm{Pr}_{2}$ & $\mathrm{Pr}_{3}$ & $P r_{1}$ & $\mathrm{Pr}_{2}$ & $\mathrm{Pr}_{3}$ & $P r_{1}$ & $\mathrm{Pr}_{2}$ & $\mathrm{Pr}_{3}$ & $P r_{1}$ & $\mathrm{Pr}_{2}$ & $\mathrm{Pr}_{3}$ \\
\hline 3 & 0.4753 & 0.4753 & 0.5903 & 0.2827 & 0.4760 & 0.5137 & 0.1583 & 0.3112 & 0.3549 & 0.0573 & 0.1331 & 0.1527 \\
\hline 4 & 0.2205 & 0.2205 & 0.4413 & 0.2565 & 0.5032 & 0.5362 & 0.1283 & 0.2631 & 0.3119 & 0.0717 & 0.1850 & 0.1883 \\
\hline 5 & 0.2965 & 0.3603 & 0.4531 & 0.2927 & 0.5052 & 0.5300 & 0.1794 & 0.3167 & 0.3477 & 0.0754 & 0.1594 & 0.1651 \\
\hline 6 & 0.3900 & 0.5231 & 0.5593 & 0.3163 & 0.5100 & 0.5322 & 0.2159 & 0.3585 & 0.3864 & 0.0615 & 0.1216 & 0.1386 \\
\hline 7 & 0.3771 & 0.5075 & 0.5442 & 0.3299 & 0.5113 & 0.5290 & 0.2019 & 0.3120 & 0.3506 & 0.0715 & 0.1290 & 0.1441 \\
\hline 8 & 0.3295 & 0.4238 & 0.4820 & 0.3426 & 0.5143 & 0.5303 & 0.2491 & 0.3748 & 0.3955 & 0.0907 & 0.1590 & 0.1686 \\
\hline 9 & 0.3189 & 0.4010 & 0.4605 & 0.3527 & 0.5134 & 288 & 0.2385 & 0.3477 & 0.3776 & 0.1034 & & 309 \\
\hline 10 & 0.3526 & 0.4521 & 0.4861 & 0.3601 & 0.5122 & 0.5245 & 0.2568 & 0.3658 & 0.3875 & 0.1036 & 0.1678 & 0.1751 \\
\hline 11 & 0.3738 & 0.4819 & 0.5066 & 0.3717 & 0.5172 & 0.5301 & 0.2684 & 0.3726 & 0.3940 & 0.1003 & 0.1578 & 0.1664 \\
\hline 50 & 0.4209 & 0.4786 & 0.4861 & 0.4677 & 0.5403 & 0.5428 & 0.3857 & 0.4432 & 0.4486 & 0.1669 & 0.2029 & 0.2048 \\
\hline 51 & 0.4201 & 0.4776 & 0.4844 & 0.4652 & 0.5363 & 0.5394 & 0.3875 & 0.4441 & 0.4495 & 0.1685 & 0.2029 & 0.2052 \\
\hline 100 & 0.4496 & 0.4915 & 0.4950 & 0.4876 & 0.5365 & 0.5376 & 0.4176 & 0.4597 & 0.4622 & 0.1867 & 0.2113 & 0.2122 \\
\hline 101 & 0.4504 & 0.4912 & 0.4943 & 0.4869 & 0.5334 & 0.5348 & 0.4178 & 0.4573 & 0.4601 & 0.1858 & 0.2102 & 0.2110 \\
\hline 1000 & 0.5149 & 0.5211 & 0.5212 & 0.5089 & 0.5153 & 0.5153 & 0.4585 & 0.4644 & 0.4645 & 0.2124 & 0.2160 & 0.2160 \\
\hline 1001 & 0.5160 & 0.5219 & 0.5219 & 0.5051 & 0.5117 & 0.5117 & 0.4607 & 0.4668 & 0.4668 & 0.2126 & 0.2162 & 0.2162 \\
\hline 100,000 & 0.5232 & 0.5233 & 0.5233 & 0.5064 & 0.5065 & 0.5065 & 0.4665 & 0.4665 & 0.4665 & 0.2172 & 0.2172 & 0.2172 \\
\hline
\end{tabular}


Table 8: The probability of agreement between Chamberlin-Courant and other multiwinner scoring rules, $m=5$ and $k=3$

\begin{tabular}{|c|c|c|c|c|c|c|c|c|c|c|c|c|}
\hline \multirow[t]{2}{*}{$n$} & \multicolumn{3}{|c|}{$\begin{array}{l}\text { Chamberlin-Courant } \\
\text { and } k \text {-Plurality }\end{array}$} & \multicolumn{3}{|c|}{$\begin{array}{l}\text { Chamberlin-Courant } \\
\text { and } k \text {-Borda }\end{array}$} & \multicolumn{3}{|c|}{$\begin{array}{c}\text { Chamberlin-Courant } \\
\text { and Bloc }\end{array}$} & \multicolumn{3}{|c|}{$\begin{array}{c}\text { Chamberlin-Courant and } \\
k \text {-Negative Plurality }\end{array}$} \\
\hline & $P r_{1}$ & $\mathrm{Pr}_{2}$ & $P r_{3}$ & $P r_{1}$ & $P r_{2}$ & $P r_{3}$ & $P r_{1}$ & $P r_{2}$ & $P r_{3}$ & $P r_{1}$ & $P r_{2}$ & $P r_{3}$ \\
\hline 3 & 0.4792 & 0.4792 & 0.9930 & 0.1678 & 0.5225 & 0.5764 & 0.0708 & 0.3052 & 0.4172 & 0.0437 & 0.1994 & 0.2586 \\
\hline 4 & 0.5787 & 0.5787 & 0.8690 & 0.2208 & 0.4759 & 0.5200 & 0.0729 & 0.1924 & 0.2700 & 0.0314 & 0.0914 & 0.1489 \\
\hline 5 & 0.4796 & 0.4796 & 0.7410 & 0.2054 & 0.4862 & 0.5236 & 0.0777 & 0.2258 & 0.2862 & 0.0430 & 0.1367 & 0.1664 \\
\hline 6 & 0.3465 & 0.3465 & 0.6734 & 0.1919 & 0.4892 & 0.5200 & 0.0811 & 0.2459 & 0.3144 & 0.0591 & 0.1950 & 0.2070 \\
\hline 7 & 0.3582 & 0.3942 & 0.6613 & 0.2121 & 0.4822 & 0.5068 & 0.0805 & 0.2112 & 0.2458 & 0.0679 & 0.1895 & 0.2005 \\
\hline 8 & 0.4335 & 0.5298 & 0.7156 & 0.2325 & 0.4803 & 0.5025 & 0.1010 & 0.2429 & 0.2867 & 0.0628 & 0.1577 & 0.1782 \\
\hline 9 & 0.4620 & 0.5873 & 0.7099 & 0.2428 & 0.4795 & 0.4991 & 0.1005 & 0.2278 & 0.2639 & 0.0609 & 0.1479 & 0.1658 \\
\hline 10 & 0.4372 & 0.5533 & 0.6730 & 0.2496 & 0.4737 & 0.4886 & 0.1065 & 0.2325 & 0.2617 & 0.0731 & 0.1673 & 0.1786 \\
\hline 11 & 0.4082 & 0.5093 & 0.6487 & 0.2619 & 0.4773 & 0.4935 & 0.1157 & 0.2376 & 0.2694 & 0.0827 & 0.1798 & 0.1887 \\
\hline 50 & 0.5214 & 0.6173 & 0.6395 & 0.3654 & 0.4641 & 0.4668 & 0.1878 & 0.2494 & 0.2566 & 0.1316 & 0.1793 & 0.1819 \\
\hline 51 & 0.5169 & 0.6111 & 0.6343 & 0.3627 & 0.4617 & 0.4646 & 0.1874 & 0.2493 & 0.2558 & 0.1299 & 0.1778 & 0.1801 \\
\hline 100 & 0.5593 & 0.6295 & 0.6389 & 0.3866 & 0.4533 & 0.4545 & 0.2090 & 0.2521 & 0.2553 & 0.1470 & 0.1801 & 0.1813 \\
\hline 101 & 0.5622 & 0.6327 & 0.6429 & 0.3869 & 0.4534 & 0.4544 & 0.2105 & 0.2545 & 0.2575 & 0.1474 & 0.1807 & 0.1816 \\
\hline 1000 & 0.6407 & 0.6513 & 0.6514 & 0.4159 & 0.4239 & 0.4239 & 0.2470 & 0.2530 & 0.2532 & 0.1788 & 0.1833 & 0.1834 \\
\hline 1001 & 0.6392 & 0.6508 & 0.6508 & 0.4170 & 0.4258 & 0.4258 & 0.2479 & 0.2540 & 0.2540 & 0.1777 & 0.1828 & 0.1828 \\
\hline 100,000 & 0.6503 & 0.6504 & 0.6504 & 0.4181 & 0.4182 & 0.4182 & 0.2525 & 0.2525 & 0.2525 & 0.1806 & 0.1806 & 0.1806 \\
\hline
\end{tabular}

Table 9: The probability of agreement between Chamberlin-Courant and other multiwinner scoring rules, $m=6$ and $k=2$

\begin{tabular}{|c|c|c|c|c|c|c|c|c|c|c|c|c|}
\hline \multirow[t]{2}{*}{$n$} & \multicolumn{3}{|c|}{$\begin{array}{c}\text { Chamberlin-Courant } \\
\text { and } k \text {-Plurality }\end{array}$} & \multicolumn{3}{|c|}{$\begin{array}{c}\text { Chamberlin-Courant } \\
\text { and } k \text {-Borda }\end{array}$} & \multicolumn{3}{|c|}{$\begin{array}{c}\text { Chamberlin-Courant } \\
\text { and Bloc }\end{array}$} & \multicolumn{3}{|c|}{$\begin{array}{c}\text { Chamberlin-Courant and } \\
k \text {-Negative Plurality }\end{array}$} \\
\hline & $P r_{1}$ & $P r_{2}$ & $P r_{3}$ & $P r_{1}$ & $P r_{2}$ & $P r_{3}$ & $P r_{1}$ & $P r_{2}$ & $P r_{3}$ & $P r_{1}$ & $P r_{2}$ & $P r_{3}$ \\
\hline 3 & 0.4151 & 0.4151 & 0.4947 & 0.2376 & 0.4077 & 0.4315 & 0.1240 & 0.2458 & 0.2820 & 0.0000 & 0.0000 & 0.0061 \\
\hline 4 & 0.1626 & 0.1626 & 0.3178 & 0.2257 & 0.4395 & 0.4604 & 0.1472 & 0.2890 & 0.3212 & 0.0225 & 0.0545 & 0.0571 \\
\hline 5 & 0.2118 & 0.2571 & 0.3167 & 0.2500 & 0.4416 & 0.4585 & 0.1364 & 0.2267 & 0.2695 & 0.0412 & 0.0900 & 0.0923 \\
\hline 6 & 0.2968 & 0.3987 & 0.4175 & 0.2738 & 0.4461 & 0.4595 & 0.2121 & 0.3328 & 0.3476 & 0.0531 & 0.1056 & 0.1068 \\
\hline 7 & 0.3124 & 0.4305 & 0.4455 & 0.2877 & 0.4476 & 0.4605 & 0.1999 & 0.2999 & 0.3287 & 0.0540 & 0.0994 & 0.1012 \\
\hline 8 & 0.2628 & 0.3534 & 0.3799 & 0.2963 & 0.4450 & 0.4570 & 0.2048 & 0.2959 & 0.3176 & 0.0463 & 0.0810 & 0.0851 \\
\hline 9 & 0.2366 & 0.3058 & 0.3406 & 0.3023 & 0.4422 & 0.4516 & 0.2354 & 0.3327 & 0.3481 & 0.0468 & 0.0781 & 0.0831 \\
\hline 10 & 0.2445 & 0.3136 & 0.3396 & 0.3136 & 0.4485 & 0.4574 & 0.2302 & 0.3165 & 0.3362 & 0.0533 & 0.0868 & 0.0926 \\
\hline 11 & 0.2711 & 0.3502 & 0.3664 & 0.3247 & 0.4528 & 0.4606 & 0.2391 & 0.3217 & 0.3385 & 0.0625 & 0.0983 & 0.1027 \\
\hline 50 & 0.3094 & 0.3540 & 0.3585 & 0.4069 & 0.4696 & 0.4716 & 0.3427 & 0.3918 & 0.3965 & 0.1104 & 0.1339 & 0.1347 \\
\hline 51 & 0.3123 & 0.3553 & 0.3597 & 0.4096 & 0.4704 & 0.4721 & 0.3453 & 0.3954 & 0.3997 & 0.1083 & 0.1310 & 0.1318 \\
\hline 100 & 0.3291 & 0.3621 & 0.3647 & 0.4321 & 0.4768 & 0.4776 & 0.3728 & 0.4098 & 0.4124 & 0.1235 & 0.1405 & 0.1413 \\
\hline 101 & 0.3239 & 0.3570 & 0.3589 & 0.4277 & 0.4724 & 0.4734 & 0.3735 & 0.4113 & 0.4141 & 0.1236 & 0.1410 & 0.1417 \\
\hline 1000 & 0.3711 & 0.3803 & 0.3808 & 0.4683 & 0.4796 & 0.4796 & 0.4302 & 0.4405 & 0.4408 & 0.1550 & 0.1600 & 0.1601 \\
\hline 1001 & 0.3739 & 0.3833 & 0.3834 & 0.4734 & 0.4850 & 0.4850 & 0.4338 & 0.4443 & 0.4444 & 0.1557 & 0.1608 & 0.1608 \\
\hline 100,000 & 0.3868 & 0.3869 & 0.3869 & 0.4713 & 0.4714 & 0.4714 & 0.4435 & 0.4436 & 0.4436 & 0.1593 & 0.1593 & 0.1593 \\
\hline
\end{tabular}


Table 10: The probability of agreement between Chamberlin-Courant and other multiwinner scoring rules, $m=6$ and $k=3$

\begin{tabular}{|c|c|c|c|c|c|c|c|c|c|c|c|c|}
\hline \multirow[t]{2}{*}{$n$} & \multicolumn{3}{|c|}{$\begin{array}{l}\text { Chamberlin-Courant } \\
\text { and } k \text {-Plurality }\end{array}$} & \multicolumn{3}{|c|}{$\begin{array}{l}\text { Chamberlin-Courant } \\
\text { and } k \text {-Borda }\end{array}$} & \multicolumn{3}{|c|}{$\begin{array}{l}\text { Chamberlin-Courant } \\
\text { and Bloc }\end{array}$} & \multicolumn{3}{|c|}{$\begin{array}{c}\text { Chamberlin-Courant and } \\
k \text {-Negative Plurality }\end{array}$} \\
\hline & $P r_{1}$ & $P r_{2}$ & $P r_{3}$ & $P r_{1}$ & $P r_{2}$ & $P r_{3}$ & $P r_{1}$ & $P r_{2}$ & $P r_{3}$ & $P r_{1}$ & $P r_{2}$ & $P r_{3}$ \\
\hline 3 & 0.5577 & 0.5577 & 1.0000 & 0.1424 & 0.4211 & 0.4696 & 0.0622 & 0.2442 & 0.3440 & 0.0280 & 0.1185 & 0.1185 \\
\hline 4 & 0.5513 & 0.5513 & 0.7556 & 0.1559 & 0.3589 & 0.3873 & 0.0370 & 0.1013 & 0.1605 & 0.0332 & 0.1048 & 0.1144 \\
\hline 5 & 0.3829 & 0.3829 & 0.5551 & 0.1379 & 0.3678 & 0.3907 & 0.0577 & 0.1866 & 0.2224 & 0.0230 & 0.0800 & 0.0875 \\
\hline 6 & 0.2308 & 0.2308 & 0.4537 & 0.1326 & 0.3710 & 0.3876 & 0.0435 & 0.1405 & 0.1933 & 0.0133 & 0.0494 & 0.0625 \\
\hline 7 & 0.2186 & 0.2512 & 0.4206 & 0.1464 & 0.3665 & 0.3815 & 0.0633 & 0.1816 & 0.2130 & 0.0171 & 0.0532 & 0.0644 \\
\hline 8 & 0.2889 & 0.3856 & 0.4941 & 0.1583 & 0.3667 & 0.3782 & 0.0605 & 0.1588 & 0.1838 & 0.0267 & 0.0766 & 0.0827 \\
\hline 9 & 0.3243 & 0.4606 & 0.5206 & 0.1675 & 0.3608 & 0.3706 & 0.0735 & 0.1805 & 0.2119 & 0.0331 & 0.0885 & 0.0927 \\
\hline 10 & 0.3164 & 0.4508 & 0.4996 & 0.1736 & 0.3578 & 0.3667 & 0.0736 & 0.1679 & 0.1919 & 0.0354 & 0.0889 & 0.0915 \\
\hline 11 & 0.2797 & 0.3887 & 0.4473 & 0.1785 & 0.3516 & 0.3596 & 0.0815 & 0.1767 & 0.1970 & 0.0329 & 0.0756 & 0.0793 \\
\hline 50 & 0.3364 & 0.4124 & 0.4236 & 0.2673 & 0.3486 & 0.3501 & 0.1509 & 0.2024 & 0.2080 & 0.0652 & 0.0901 & 0.0911 \\
\hline 51 & 0.3343 & 0.4107 & 0.4207 & 0.2665 & 0.3441 & 0.3462 & 0.1523 & 0.2023 & 0.2080 & 0.0642 & 0.0886 & 0.0898 \\
\hline 100 & 0.3612 & 0.4195 & 0.4256 & 0.2886 & 0.3448 & 0.3456 & 0.1748 & 0.2141 & 0.2169 & 0.0745 & 0.0937 & 0.0944 \\
\hline 101 & 0.3578 & 0.4143 & 0.4198 & 0.2925 & 0.3495 & 0.3509 & 0.1769 & 0.2153 & 0.2189 & 0.0777 & 0.0982 & 0.0988 \\
\hline 1000 & 0.4168 & 0.4338 & 0.4341 & 0.3282 & 0.3427 & 0.3427 & 0.2214 & 0.2322 & 0.2323 & 0.0975 & 0.1035 & 0.1035 \\
\hline 1001 & 0.4182 & 0.4340 & 0.4345 & 0.3271 & 0.3406 & 0.3406 & 0.2209 & 0.2309 & 0.2311 & 0.0980 & 0.1036 & 0.1037 \\
\hline 100,000 & 0.4355 & 0.4357 & 0.4357 & 0.3282 & 0.3284 & 0.3284 & 0.2271 & 0.2273 & 0.2273 & 0.1031 & 0.1033 & 0.1033 \\
\hline
\end{tabular}

Table 11: The probability of agreement between Chamberlin-Courant and other multiwinner scoring rules, $m=6$ and $k=4$

\begin{tabular}{|c|c|c|c|c|c|c|c|c|c|c|c|c|}
\hline \multirow[t]{2}{*}{$n$} & \multicolumn{3}{|c|}{$\begin{array}{l}\text { Chamberlin-Courant } \\
\text { and } k \text {-Plurality }\end{array}$} & \multicolumn{3}{|c|}{$\begin{array}{c}\text { Chamberlin-Courant } \\
\text { and } k \text {-Borda }\end{array}$} & \multicolumn{3}{|c|}{$\begin{array}{c}\text { Chamberlin-Courant } \\
\text { and Bloc }\end{array}$} & \multicolumn{3}{|c|}{$\begin{array}{c}\text { Chamberlin-Courant and } \\
k \text {-Negative Plurality }\end{array}$} \\
\hline & $P r_{1}$ & $P r_{2}$ & $P r_{3}$ & $P r_{1}$ & $P r_{2}$ & $\mathrm{Pr}_{3}$ & $P r_{1}$ & $P r_{2}$ & $\mathrm{Pr}_{3}$ & $P r_{1}$ & $P r_{2}$ & $\mathrm{Pr}_{3}$ \\
\hline 3 & 0.0000 & 0.0000 & 1.0000 & 0.0000 & 0.6164 & 0.7164 & 0.0000 & 0.3021 & 0.4494 & 0.0000 & 0.2134 & 0.3319 \\
\hline 4 & 0.2763 & 0.2763 & 0.9946 & 0.0712 & 0.5046 & 0.5640 & 0.0205 & 0.2072 & 0.3155 & 0.0063 & 0.0648 & 0.1450 \\
\hline 5 & 0.4669 & 0.4669 & 0.9378 & 0.1260 & 0.4385 & 0.4769 & 0.0303 & 0.1397 & 0.2447 & 0.0180 & 0.0866 & 0.1121 \\
\hline 6 & 0.5007 & 0.5007 & 0.8492 & 0.1458 & 0.4192 & 0.4485 & 0.0498 & 0.1810 & 0.2482 & 0.0329 & 0.1301 & 0.1426 \\
\hline 7 & 0.4487 & 0.4487 & 0.7607 & 0.1417 & 0.4230 & 0.4506 & 0.0426 & 0.1603 & 0.2253 & 0.0364 & 0.1508 & 0.1618 \\
\hline 8 & 0.3699 & 0.3699 & 0.7001 & 0.1365 & 0.4215 & 0.4429 & 0.0428 & 0.1616 & 0.2266 & 0.0305 & 0.1276 & 0.1436 \\
\hline 9 & 0.3347 & 0.3469 & 0.6728 & 0.1389 & 0.4118 & 0.4296 & 0.0479 & 0.1700 & 0.2161 & 0.0260 & 0.1013 & 0.1187 \\
\hline 10 & 0.3628 & 0.4091 & 0.6901 & 0.1479 & 0.4050 & 0.4195 & 0.0477 & 0.1576 & 0.1958 & 0.0297 & 0.1031 & 0.1176 \\
\hline 11 & 0.4029 & 0.4867 & 0.7040 & 0.1558 & 0.3973 & 0.4118 & 0.0528 & 0.1571 & 0.1975 & 0.0358 & 0.1138 & 0.1227 \\
\hline 50 & 0.4581 & 0.5718 & 0.6062 & 0.2512 & 0.3594 & 0.3616 & 0.1022 & 0.1556 & 0.1630 & 0.0719 & 0.1130 & 0.1149 \\
\hline 51 & 0.4580 & 0.5719 & 0.6076 & 0.2504 & 0.3556 & 0.3580 & 0.1002 & 0.1534 & 0.1604 & 0.0714 & 0.1118 & 0.1140 \\
\hline 100 & 0.4932 & 0.5849 & 0.6007 & 0.2733 & 0.3495 & 0.3503 & 0.1159 & 0.1561 & 0.1601 & 0.0829 & 0.1129 & 0.1138 \\
\hline 101 & 0.4950 & 0.5860 & 0.6027 & 0.2761 & 0.3510 & 0.3517 & 0.1173 & 0.1572 & 0.1611 & 0.0832 & 0.1147 & 0.1154 \\
\hline 1000 & 0.5761 & 0.6038 & 0.6049 & 0.3193 & 0.3388 & 0.3388 & 0.1476 & 0.1586 & 0.1586 & 0.1100 & 0.1188 & 0.1188 \\
\hline 1001 & 0.5772 & 0.6034 & 0.6038 & 0.3180 & 0.3359 & 0.3361 & 0.1490 & 0.1592 & 0.1595 & 0.1073 & 0.1155 & 0.1155 \\
\hline 100,000 & 0.6050 & 0.6052 & 0.6052 & 0.3229 & 0.3230 & 0.3230 & 0.1566 & 0.1566 & 0.1566 & 0.1172 & 0.1173 & 0.1173 \\
\hline
\end{tabular}




\section{References}

Aziz, H., Brill, M., Conitzer, V., Elkind, E., Freeman, R., and Walsh, T. (2017) Justified representation in approval-based committee voting. Social Choice and Welfare, 48(2): 461485.

Aziz, H., Gaspers, S., Mattei, N., Narodytska, N., and Walsh, T. (2013) Ties matter: Complexity of manipulation when tie-breaking with a random vote. In Proc. of 27th AAAI Conference, pages 74-80. AAAI Press.

Barberà, S., and Coelho, D. (2008) How to choose a non-controversial list with $k$ names. Social Choice and Welfare, 31: 79-96.

Betzler, N., Slinko, A., and Uhlmann, J. (2013) On the computation of fully proportional representation. Journal of Artificial Intelligence Research, 47: 475-519.

Bock, H., Dayb, W., and McMorris, F. (1998) Consensus rules for committee elections. Mathematical Social Sciences, 35(3): 219-232.

Brams, S.J. (2008) Mathematics and Democracy: Designing Better Voting and Fair-Division Procedures. Princeton University Press.

Brams, S.J., Kilgour, D.M., and Sanver, M.R. (2005) A minimax procedure for electing committees. Public Choice, 132(34): 401-420.

Brams, S.J., Kilgour, D.M., and Sanver, M.R. (2006) How to elect a representative committee using approval balloting. In: Simeone B., Pukelsheim F., editors. Mathematics and Democracy. Springer; Berlin. pp. 83-95. (Studies in Choice and Welfare).

Bruns, W., Ichim, B., Römer, T., Sieg, R., and Söger, C. (2017) Normaliz: Algorithms for rational cones and affine monoids. Available at http://normaliz.uos.de.

Bruns, W., Ichim, B., and Söger, C. (2017) Computations of volumes and Ehrhart series in four candidates elections. Working paper, Cornell University Library.

Cervone, D., Gehrlein, W.V., and Zwicker, W. (2005) Which scoring rule maximizes Condorcet efficiency under IAC? Theory and Decision, 58: 145-185.

Chamberlin, J.R., and Courant, P.N. (1983). Representative Deliberations and Representative Decisions: Proportional Representation and the Borda Rule. The American Political Science Review, 77(3): 718-733.

Courtin, S., Martin, M., and Moyouwou, I. (2015) The $q$-majority efficiency of positional rules. Theory and Decision, 79: 31-49.

Debord, B. (1993) Prudent $k$-choice functions: Properties and algorithms. Mathematical Social Sciences, 26: 63-77.

Diss, M. (2015) Strategic manipulability of self-selective social choice rules. Annals of Operations Research, 229: 347-376. 
Diss, M., and Doghmi, A. (2016) Multi-winner scoring election methods: Condorcet consistency and paradoxes. Public Choice, 169: 97-116

Diss, M., and Gehrlein, W.V. (2015) The true impact of voting rule selection on Condorcet efficiency. Economics Bulletin, 35: 2418-2426.

Diss, M., and Gehrlein, W.V. (2012) Borda's paradox with weighted scoring rules. Social Choice and Welfare, 38: 121-136.

Diss, M., Louichi, A., Merlin, V., and Smaoui, H. (2012) An example of probability computations under the IAC assumption: The stability of scoring rules. Mathematical Social Sciences, 64: 57-66.

Dodgson C.L (1884) The principles of parliamentary representation. Harrison and Sons Publisher, London.

Dodgson C.L (1876) A Method of Taking Votes on More than Two Issues. Clarendon Press, Oxford.

Droop H. (1881) On methods of electing representatives. Journal of the Statistical Society of London, 44(2): 141-202.

Dummett, M. (1984) Voting Procedures. Oxford, Clarendon Press.

Elkind, E., Faliszewski P., Skowron, P., and Slinko, A. (2017) Properties of multiwinner voting rules. Social Choice and Welfare, 48(3): 599-632.

Elkind, E., Lang, J., and Saffidine, A. (2011) Choosing collectively optimal sets of alternatives based on the Condorcet criterion. Proceedings IJCAI11, pp. 186-191, https: //www.ijcai.org/Proceedings/11/Papers/042.pdf.

Elkind, E., Lang, J., and Saffidine, A. (2015) Condorcet winning sets. Social Choice and Welfare, 44(3): 493-517.

Franz, M. (2016) Convex - a Maple package for convex geometry, version 1.2 available at http: //www-home.math.uwo.ca/ mfranz/convex/.

Faliszewski, P., Skowron, P., Slinko, A., and Talmon, N. (2017) Multiwinner Rules on Paths From $k$-Borda to Chamberlin-Courant. Proceedings IJCAI17, pp. 192-198, https://doi . org/10.24963/ijcai.2017/28.

Fishburn, P.C. (1981) An analysis of simple voting systems for electing committees. SIAM Journal on Applied Mathematics, 41: 499-502.

Gehrlein, W.V. (1985) The Condorcet criterion and committee selection. Mathematical Social Sciences, 10: 199-209.

Gehrlein, W.V., and Fishburn, P.C. (1976) The probability of the paradox of voting: A computable solution. Journal of Economic Theory, 13: 14-25. 
Gehrlein, W.V., and Lepelley, D. (2017) Elections, Voting Rules and Paradoxical Outcomes. Springer-Verlag.

Gehrlein, W.V., and Lepelley, D. (2011) Voting paradoxes and group coherence. Springer, Berlin/Heidelberg.

Gehrlein, W.V., Lepelley, D., and Plassmann, F. (2017) An Evaluation of the Benefit of Using Two-Stage Election Procedures. Homo Oeconomicus, forthcoming, https://doi . org/10.1007/s41412-017-0055-2.

Gehrlein, W.V., Lepelley, D., and Moyouwou, I. (2016) A note on Approval Voting and electing the Condorcet loser. Mathematical Social Sciences, 80: 115-122.

Gehrlein, W.V., Lepelley, D., and Moyouwou, I. (2015) Voters' preference diversity, concepts of agreement and Condorcet's paradox. Quality \& Quantity, 49(6): 2345-2368.

Kamwa, E. (2017) On stable rules for selecting committees. Journal of Mathematical Economics, 70: 36-44.

Kamwa, E., and Merlin, V. (2014) Some remarks on the Chamberlin-Count rule. Mimeo, Université de Caen.

Kamwa, E., and Merlin, V. (2015) Scoring rules over subsets of alternatives: Consistency and paradoxes. Journal of Mathematical Economics, 61: 130-138.

Kamwa, E., and Merlin, V. (2018) Coincidence of Condorcet Committees. Social Choice and Welfare, 50(1): 171-189.

Kamwa, E., and Valognes, F. (2017) Scoring rules and preference restrictions: The Strong Borda Paradox revisited. Revue d'Economie Politique, 127(3): 375-395.

Kaymak, B., and Sanver, M.R. (2003) Sets of alternatives as Condorcet winners. Social Choice and Welfare, 20: 477-494.

Kilgour, M., and Marshall, E. (2012) Approval balloting for fixed-size committees. In Felsenthal, Dan S., and Machover, M. editors, Electoral Systems: Paradoxes, Assumptions, and Procedures, 305-326. Springer Heidelberg.

Kilgour, D.M., Brams, S.J., and Sanver, M.R. (2006) How to elect a representative committee using approval balloting. In B. Simeone and F. Pukelsheim, editors, Mathematics and Democracy: Recent Advances in Voting Systems and Collective Choice. Springer Heidelberg.

Lepelley, D., Moyouwou, I., and Smaoui, H. (2018) Monotonicity paradoxes in threecandidate elections using scoring elimination rules. Social Choice and Welfare, 50 (1): $1-33$.

Lepelley, D., Louichi, A., and Smaoui, H. (2008) On Ehrhart polynomials and probability calculations in voting theory. Social Choice and Welfare, 30: 363-383. 
Lu, T., and Boutilier, C. (2011) Budgeted social choice: From consensus to personalized decision making. In Proceedings of IJCAI-11: 280-286.

Mattei, N., Narodytska, N., and Walsh T. (2014) How hard is it to control an election by breaking ties? In Proc. of 21st ECAI, pages 1067-1068.

Moyouwou, I., and Tchantcho, H. (2017) A note on Approval Voting and electing the Condorcet loser. Mathematical Social Sciences, 89: 70-82.

Obraztsova, S., Elkind, E., and Hazon, N. (2011) Ties matter: Complexity of voting manipulation revisited. In Proc. of 10th AAMAS Conference, pages 71-78.

Potthoff, R.F., and Brams S.J. (1998) Proportional representation: Broadening the options. Journal of Theoritical Politics 10(2): 147-178.

Procaccia, A.D., Rosenschein, J.S., and Zohar, A. (2008) On the complexity of achieving proportional representation. Social Choice and Welfare, 30: 353-362.

Ratliff, T.C. (2003) Some startling inconsistencies when electing committees. Social Choice and Welfare, 21: 433-454.

Ratliff, T.C. (2006) Selecting committees. Public Choice, 126: 343-355.

Skowron, P., Faliszewski, P., and Slinko, A. (2016) Axiomatic Characterization of Committee Scoring Rules. Preprint arXiv: 1604.01529

Skowron, P., Faliszewski, P., and Slinko, A. (2013) Achieving fully proportional representation: Approximability results. Preprint arXiv: 1312.4026.

Skowron, P., Yu, L., Faliszewski, P., and Elkind, E. (2015) The complexity of fully proportional representation for single-crossing electorates. Theoretical Computer Science, 569: $43-57$.

Smaoui, H., Lepelley, D., and Moyouwou, I. (2016) Borda elimination rule and monotonicity paradoxes in three-candidate elections. Economics Bulletin, 36(3): 1722-1728.

Sterne, S. (1871) On representative government and personal representation. Philadelphia: J.B. Lippincott.

Verdoolaege, S., Woods, K.M., Bruynooghe, M., and Cools, R. (2005) Computation and Manipulation of Enumerators of Integer Projections of Parametric Polytopes. Technical Report CW 392, Katholieke Universiteit Leuven.

Wilson, M.C., and Pritchard, G. (2007) Probability calculations under the IAC hypothesis. Mathematical Social Sciences, 54: 244-256.

Young, H.P (1974) An axiomatization of the Borda rule. Journal of Economic Theory, 9: 43-52. 